MATHEMATICS OF COMPUTATION

Volume 72 , Number 242, Pages 541-576

S 0025-5718(02)01441-2

Article electronically published on June 28, 2002

\title{
A MIXED MULTISCALE FINITE ELEMENT METHOD FOR ELLIPTIC PROBLEMS WITH OSCILLATING COEFFICIENTS
}

\author{
ZHIMING CHEN AND THOMAS Y. HOU
}

\begin{abstract}
The recently introduced multiscale finite element method for solving elliptic equations with oscillating coefficients is designed to capture the large-scale structure of the solutions without resolving all the fine-scale structures. Motivated by the numerical simulation of flow transport in highly heterogeneous porous media, we propose a mixed multiscale finite element method with an over-sampling technique for solving second order elliptic equations with rapidly oscillating coefficients. The multiscale finite element bases are constructed by locally solving Neumann boundary value problems. We provide a detailed convergence analysis of the method under the assumption that the oscillating coefficients are locally periodic. While such a simplifying assumption is not required by our method, it allows us to use homogenization theory to obtain the asymptotic structure of the solutions. Numerical experiments are carried out for flow transport in a porous medium with a random log-normal relative permeability to demonstrate the efficiency and accuracy of the proposed method.
\end{abstract}

\section{INTRODUCTION}

Many problems of fundamental and practical importance in science and engineering have multiple-scale solutions. Typical examples include composite materials with fine micro-structures and highly heterogeneous porous media. The direct numerical simulation of problems involving multiscale solutions is difficult, due to the requisite of tremendous amount of computer memory and CPU time, which can easily exceed the limit of today's computer resources. On the other hand, in practice, it is often sufficient to predict the large scale solutions to a certain accuracy. Thus, various methods of upscaling or homogenization have been developed which replace the governing equations with multiscale solutions by the homogenized equations, whose solutions can be resolved on a coarse-scale mesh.

The recently developed multiscale finite element method [18, 19], 13] provides an effective way to capture the large-scale structures of the solutions on a coarse mesh. The central idea of the method is to incorporate the local small-scale information of the leading-order differential operator into the finite element bases. It is through these multiscale bases and the finite element formulation that the effect of

Received by the editor March 21, 2000 and, in revised form, July 10, 2000 and May 29, 2001.

2000 Mathematics Subject Classification. Primary 65F10, 65F30.

The first author was supported in part by China NSF under the grants 19771080 and 10025102 and by China MOS under the grant G1999032804.

The second author was supported in part by NSF under the grant DMS-0073916 and by ARO under the grant DAAD19-99-1-0141. 
small scales on the large scales is correctly captured. Apart from the computational advantages, such as saving in computer memory and good parallel efficiency [18] of the multiscale finite element method, we stress that the real significance of the method lies in its ability to solve the problems in coarse meshes. This is particularly advantageous when multiple simulations or realizations are necessary due to changes of boundary conditions or source functions for given fine microstructures of composite materials or highly heterogeneous permeability of porous media. Other relevant works on constructing special finite element bases can be found in [3] for layered microstructures and in [6] for convection-dominated diffusion problems.

The motivation of this paper is to explore the possibility of applying the multiscale finite element method to numerical computation of flow transport in highly heterogeneous porous media. In its simplest form, neglecting the effect of gravity, compressibility, capillary pressure, and considering constant porosity and unit mobility, the governing equations for the flow transport can be described by the following partial differential equations (see [21, [31, and [12]):

$$
\begin{aligned}
& \operatorname{div}(\mathcal{K}(x) \nabla p)=0 \\
& \frac{\partial S}{\partial t}+\mathbf{v} \cdot \nabla S=0
\end{aligned}
$$

where $p$ is the pressure, $S$ is the water saturation, $\mathcal{K}(x)=\left(\mathcal{K}_{i j}(x)\right)$ is the relative permeability tensor, and $\mathbf{v}=-\mathcal{K}(x) \nabla p$ is the Darcy velocity. The highly heterogeneous properties of the medium are built into the permeability tensor $\mathcal{K}(x)$, which is generated through the use of sophisticated geological and geostatistical modeling tools. The detailed structure of the permeability coefficients makes the direct simulation of the above model infeasible. For example, it is common in real simulations to use millions of grid blocks, with each block having a dimension of tens of meters, whereas the permeability measured from cores is at a scale of centimeters 24]. This gives more than $10^{5}$ degrees of freedom per spatial dimension in the computation. This makes a direct simulation to resolve all small scales prohibitive even with today's most powerful supercomputers. On the other hand, from an engineering perspective, it is often sufficient to predict the macroscopic properties of the solutions. Thus it is highly desirable to derive effective coarse grid models to capture the correct large solution without resolving the small-scale features. Numerical upscaling is one of the commonly used approaches in practice. There are extensive studies in the literature on the numerical upscaling of two-phase flows through porous media, see, e.g., [1, 21], [11, 14], 30].

Mixed finite element approximations for second order elliptic problems, which approximate the source variable and flux simultaneously, have been studied by many authors (cf., e.g., [27] and the book [5]). The local conservation of velocity flux is an important property in the mixed finite element methods. The violation of this local conservation property will lead to leakage of velocity flux. This will deteriorate the accuracy of the numerical solution for long-time computations. This is the reason why mixed finite element methods are attractive for porous medium simulations [28] (see also the numerical results in $\$ 5.1$ of this paper). In this paper, we first propose and analyze a mixed multiscale finite element method with an over-sampling technique for solving elliptic equations with oscillating coefficients, and then apply the method to compute the above flow model (1.1)-(1.2). The use of the over-sampling technique is crucial in eliminating large resonance errors from 
the element boundaries. This point has already been well demonstrated in the previous study [18, 13] for the displacement multiscale finite element method. We will demonstrate that this technique applies also to the mixed multiscale finite element method. Our computational results show that the mixed multiscale method with the over-sampling technique gives more accurate results than the corresponding method without such technique.

We have performed careful numerical experiments to validate our mixed multiscale finite element method. To test how well our method works in a realistic application, we apply our method to a flow in porous media with a random log-normal relative permeability tensor. This is one of the practical benchmark test cases for oil recovery problems. Our computational results demonstrate convincingly the importance of the local conservation property in the flow simulation. When the local conservation property is not satisfied, the fractional flow curve deviates significantly from that obtained from the well-resolved mixed finite element calculation after a short time. The mixed multiscale finite element method, on the other hand, provides an accurate numerical approximation even on a coarse grid, with accuracy comparable to that obtained using the standard fine-mesh mixed finite element method. Finally, we compare the performance of the mixed multiscale method studied in this paper with the displacement multiscale finite element method studied in [18], 19], 13] when applied to the flow transport problem in heterogeneous porous media. The two methods give similar results at an early stage. But the fractional flow curve obtained by the displacement multiscale method deviates significantly from that obtained by the well-resolved calculation after a short time, clearly suffering from the violation of the local conservation property. The mixed multiscale method gives a more accurate solution than the displacement multiscale method for long-time calculations, due to the inherent local conservation property of the mixed multiscale method.

The ultimate goal of this study is to produce an effective coarse grid model for the two-phase flow with heterogeneous porous media. To this end, we need to upscale the saturation equation. Without capillary pressure, the saturation equation is hyperbolic. The effective equation is difficult to derive and has a nonlocal memory effect [29]. Using an upscaling technique recently developed in [14] for the saturation equation, we show how the proposed mixed multiscale finite element method leads to a complete coarse grid algorithm. Our numerical results demonstrate convincingly that the fractional flow curve obtained from the resulting coarse grid model gives a very good approximation to that obtained from the fine grid calculation. Typically, many realizations on the same microstructure (permeability field) are made due to changes in boundary conditions and source fields. In such a case, the multiscale finite element bases are only constructed once, and can be used in the subsequent computations. Thus, the coarse grid model offers substantial saving in both memory and computing time. The saving could be as large as a factor of 10,000 if one can scale up by a factor of 10 in each space dimension (three space dimensions plus time).

The outline of the paper is as follows. In $\$ 2$ we introduce the mixed multiscale finite element methods and present the main convergence results. In $\$ 3$ we review the homogenization results for elliptic problems with Neumann boundary conditions. These results are the basis of our convergence analysis. In \$4 we prove the error estimate for the mixed multiscale finite element method with over-sampling 
introduced in $\S 2.3$. The analysis depends on an abstract formulation for nonconforming mixed finite element methods, the homogenization theory, and a technique of "freezing coefficients" to deal with the local periodic coefficients. In \$5 we apply our method to simulate the flow transport model (1.1)-(1.2) for a practical random log-normal permeability.

\section{Mixed multiscale finite element methods}

2.1. Notation and background. Let $\Omega$ be a polyhedral domain in $\mathbf{R}^{d}(d=2,3)$ with boundary $\partial \Omega$ whose unit outer normal is denoted by $\mathbf{n}$. Let $D \subseteq \Omega$ be a domain in $\mathbf{R}^{d}$ with the Lipschitz boundary $\Gamma$. For each integer $m \geq 0$ and $1 \leq p \leq \infty$, we denote by $W^{m, p}(D)$ the standard Sobolev space of real functions having all their weak derivatives of order up to $m$ in the Lebesgue space $L^{p}(D)$. The norm and the seminorm of $W^{m, p}(D)$ will be denoted by $\|\cdot\|_{m, p, D}$ and $|\cdot|_{m, p, D}$, respectively. As usual, when $p=2, W^{m, 2}(D)$ is denoted by $H^{m}(D)$ with the norm $\|\cdot\|_{m, D}$ and the seminorm $|\cdot|_{m, D}$. In the following, we let $V(D)$ denote the subspace of $H^{1}(D)$ whose functions have zero average over $D$.

We consider the following second order elliptic equations with locally periodic coefficients [4]:

$$
\begin{aligned}
L_{\varepsilon} u_{\varepsilon}:=-\frac{\partial}{\partial x_{i}}\left(a_{i j}\left(x, \frac{x}{\varepsilon}\right) \frac{\partial u_{\varepsilon}}{\partial x_{j}}\right)=f & \text { in } \quad \Omega, \\
-a\left(x, \frac{x}{\varepsilon}\right) \nabla u_{\varepsilon} \cdot \mathbf{n}=g & \text { on } \quad \partial \Omega .
\end{aligned}
$$

In this paper the usual Einstein summation convention for repeated indices is used. Here, $\varepsilon$ is assumed to be a small parameter, and $a(x, x / \varepsilon)=\left(a_{i j}(x, x / \varepsilon)\right)$ is a symmetric matrix which satisfies the uniform ellipticity condition:

$$
\gamma|\xi|^{2} \leq a_{i j}(x, y) \xi_{i} \xi_{j} \leq \gamma^{-1}|\xi|^{2} \quad \forall \xi \in \mathbf{R}^{d}, x \in \bar{\Omega}, y \in \mathbf{R}^{d},
$$

for some positive constant $\gamma$. Furthermore, we assume that $a_{i j} \in C^{1}\left(\bar{\Omega} ; C_{p}^{1}\left(\mathbf{R}^{d}\right)\right)$, where $C_{p}^{1}\left(\mathbf{R}^{d}\right)$ stands for the collection of all $C^{1}\left(\mathbf{R}^{d}\right)$ periodic functions with respect to the unit cube $Y$.

Let $u_{0}$ be the solution of the homogenized problem of (2.1)-(2.2):

$$
\begin{aligned}
L_{0} u_{0}:=-\frac{\partial}{\partial x_{i}}\left(a_{i j}^{*}(x) \frac{\partial u_{0}}{\partial x_{j}}\right)=f & \text { in } \quad \Omega, \\
-a^{*}(x) \nabla u_{0} \cdot \mathbf{n} & =g \quad \text { on } \quad \partial \Omega,
\end{aligned}
$$

where $a^{*}(x)=\left(a_{i j}^{*}(x)\right)$ with

$$
a_{i j}^{*}(x)=\frac{1}{|Y|} \int_{Y} a_{i k}(x, y)\left(\delta_{k j}-\frac{\partial \chi^{j}}{\partial y_{k}}(x, y)\right) d y,
$$

and $\chi^{j}(x, y)$ is the periodic solution of the cell problem

$$
\frac{\partial}{\partial y_{i}}\left(a_{i k}(x, y) \frac{\partial \chi^{j}}{\partial y_{k}}(x, y)\right)=\frac{\partial}{\partial y_{i}} a_{i j}(x, y) \quad \text { in } Y, \quad \int_{Y} \chi^{j}(x, y) d y=0 .
$$

Here $\delta_{k j}$ is the Kronecker delta, i.e., $\delta_{k j}=1$ for $k=j$ and $\delta_{k j}=0$ for $k \neq j$. Note that in (2.7) $x$ plays the role of a parameter. However, since $a_{i j}(x, y)$ is differentiable in $x$, we can easily show that $\chi^{j}(x, y)$ is also differentiable in $x$. The convergence property of $u_{\varepsilon}$ to $u_{0}$ will be studied in detail in the next section. 
Throughout the paper we impose the following assumptions on the data:

(H1) $f \in H^{1}(\Omega), g=\mathbf{q}_{0} \cdot \mathbf{n}$ on $\partial \Omega$ for some $\mathbf{q}_{0} \in H^{1}(\Omega)^{d}$.

(H2) Compatibility: $\int_{\Omega} f d x=\int_{\partial \Omega} g d s$.

We remark that the assumption on the boundary value $g$ is not very restrictive. For example, in the case when $\Omega$ is a convex polygon in $\mathbf{R}^{2}$ with sides $\Gamma_{j}, 1 \leq j \leq J$, and $g \in \Pi_{j=1}^{J} H^{1 / 2}\left(\Gamma_{j}\right)$, such a flux $\mathbf{q}_{0}$ can be constructed through $\mathbf{q}_{0}=-\nabla u^{*}$ with $u^{*} \in H^{1}(\Omega)$ being the solution of the equation $-\Delta u^{*}+u^{*}=0$ in $\Omega$ with the Neumann boundary condition $\nabla u^{*} \cdot \mathbf{n}=g$ on $\partial \Omega$. The regularity results in [17] then ensure $\mathbf{q}_{0} \in H^{1}(\Omega)$. We also stress that the explicit formula of such a flux $\mathbf{q}_{0}$ is not required in the definition of our methods.

Denote by $L_{0}^{2}(\Omega)$ the subspace of $L^{2}(\Omega)$ whose functions have zero average over $\Omega$, and let $H_{0}(\operatorname{div} ; \Omega)$ be the subspace of $H(\operatorname{div} ; \Omega)$ given by

$$
H_{0}(\operatorname{div} ; \Omega)=\left\{\mathbf{v} \in L^{2}(\Omega)^{d}: \operatorname{div} \mathbf{v} \in L^{2}(\Omega), \mathbf{v} \cdot \mathbf{n}=0 \quad \text { on } \partial \Omega\right\} .
$$

The norm of $H(\operatorname{div} ; \Omega)$ will be denoted by $\|\cdot\|_{\operatorname{div}, \Omega}$. Let $\mathbf{p}_{\varepsilon}=-a(x, x / \varepsilon) \nabla u_{\varepsilon}$ and $a^{-1}(x, y)$ the inverse matrix of $a(x, y)$; then $\nabla u_{\varepsilon}=-a^{-1}(x, x / \varepsilon) \mathbf{p}_{\varepsilon}$. The mixed formulation to (2.1)-2.2) then reads as follows: find a pair $\left(\mathbf{p}_{\varepsilon}, u_{\varepsilon}\right) \in H(\operatorname{div} ; \Omega) \times$ $L_{0}^{2}(\Omega)$ such that $\mathbf{p}_{\varepsilon} \cdot \mathbf{n}=g$ on $\partial \Omega$ and

$$
\begin{aligned}
& \left(\operatorname{div} \mathbf{p}_{\varepsilon}, v\right)=(f, v) \quad \forall v \in L^{2}(\Omega), \\
& \left(a^{-1}(x, x / \varepsilon) \mathbf{p}_{\varepsilon}, \mathbf{q}\right)-\left(u_{\varepsilon}, \operatorname{div} \mathbf{q}\right)=0 \quad \forall \mathbf{q} \in H_{0}(\operatorname{div} ; \Omega) .
\end{aligned}
$$

Here $(\cdot, \cdot)$ stands for the inner product of $L^{2}(\Omega)$ or $L^{2}(\Omega)^{d}$. The existence of a unique solution of problem (2.8)-(2.9) and its equivalence to (2.1)-(2.2) follow directly from the abstract Babuska-Brezzi theory [5], [27].

Let us assume that $\mathcal{T}_{h}$ is a regular and quasi-uniform partition of $\Omega$ into simplices. For any $K \in \mathcal{T}_{h}$, let $h_{K}$ be its diameter, $|K|$ its Lebesgue measure, $\nu^{K}$ the unit outer normal to $\partial K$, and $\left\{e_{j}^{K}\right\}_{j=1}^{d+1}$ the surfaces or edges of $\partial K$ with $\left|e_{j}^{K}\right|$ being the measure of $e_{j}^{K}$. The mixed multiscale finite element method that we are going to introduce is closely related to the Raviart-Thomas elements [27]. For any $K \in \mathcal{T}_{h}$, we denote by $R T_{0}(K)$ the lowest order Raviart-Thomas approximation of $H(\operatorname{div}, K)$ :

$$
R T_{0}(K)=P_{0}(K)^{d}+\mathbf{x} P_{0}(K), \quad \mathbf{x}=\left(x_{1}, \cdots, x_{d}\right)^{\mathrm{T}} \in \mathbf{R}^{d}
$$

where $P_{0}(K)$ is the constant element space. Let $\left\{R_{i}^{K}\right\}_{i=1}^{d+1}$ be the basis of $R T_{0}(K)$ which satisfies

$$
R_{i}^{K} \cdot \nu^{K}=\left\{\begin{array}{cll}
\frac{1}{\left|e_{i}^{K}\right|} & \text { on } & e_{i}^{K} \\
0 & \text { on } & e_{j}^{K}, j \neq i
\end{array}\right.
$$

Since $\operatorname{div} R_{i}^{K}$ is constant in $K$, it follows easily from Green's formula that $\operatorname{div} R_{i}^{K}=$ $1 /|K|$. Let $W_{h} \subset H(\operatorname{div} ; \Omega)$ be the lowest order Raviart-Thomas finite element space. It is well-known that there exists an interpolation operator $r_{h}: H(\operatorname{div}, \Omega) \cap$ $H^{1}(\Omega)^{d} \rightarrow W_{h}$ such that $r_{K}:=\left.r_{h}\right|_{K}$ satisfies the relations

$$
\int_{e_{j}^{K}}\left(r_{K} \mathbf{q}-\mathbf{q}\right) \cdot \nu^{K} d s=0 \quad \forall \mathbf{q} \in H^{1}(K)^{d}, \quad j=1, \cdots, d+1
$$

and the error estimate

$$
\left\|\mathbf{q}-r_{K} \mathbf{q}\right\|_{m, K} \leq C h_{K}^{1-m}|\mathbf{q}|_{1, K} \quad \forall \mathbf{q} \in H^{1}(K)^{d}, \quad m=0,1 .
$$


Let $M_{h} \subset L_{0}^{2}(\Omega)$ be the standard piecewise constant finite element space for approximating $u_{\varepsilon}$. Then the standard mixed finite element method using the lowest order Raviart-Thomas element leads to the following discrete problem: find a pair $\left(\mathbf{p}_{h}, u_{h}\right) \in W_{h} \times M_{h}$ such that $\mathbf{p}_{h} \cdot \mathbf{n}=r_{h} \mathbf{q}_{0} \cdot \mathbf{n}$ on $\partial \Omega$ and

$$
\begin{aligned}
& \left(\operatorname{div} \mathbf{p}_{h}, v_{h}\right)=\left(f, v_{h}\right) \quad \forall v_{h} \in M_{h}, \\
& \left(a^{-1}(x, x / \varepsilon) \mathbf{p}_{h}, \mathbf{q}_{h}\right)-\left(u_{h}, \operatorname{div} \mathbf{q}_{h}\right)=0 \quad \forall \mathbf{q}_{h} \in W_{h} \cap H_{0}(\operatorname{div} ; \Omega) .
\end{aligned}
$$

The following well-known error estimate can be obtained by using the BabuskaBrezzi theory 27, [5]

$$
\left\|\mathbf{p}_{\varepsilon}-\mathbf{p}_{h}\right\|_{\operatorname{div}, \Omega}+\left\|u_{\varepsilon}-u_{h}\right\|_{0, \Omega} \leq C h\left(\left\|\mathbf{p}_{\varepsilon}\right\|_{1, \Omega}+\left\|u_{\varepsilon}\right\|_{1, \Omega}\right) .
$$

Note that since $\left\|\mathbf{p}_{\varepsilon}\right\|_{1, \Omega} \leq C \varepsilon^{-1}$ [19], the error estimate (2.12) implies that the mesh size $h$ must satisfy $h<<\varepsilon$ to obtain accurate approximations. The purpose of the mixed multiscale finite element methods to be introduced next is to remove such a strong requirement on the mesh size $h$. This is achieved by building the local small-scale information into the finite element bases.

2.2. Mixed multiscale finite element method. Recalling that $V(K)$ denotes the subspace of $H^{1}(K)$ whose functions have zero average over $K$, we define $w_{i}^{K} \in$ $V(K)$ as the solution of the following Neumann problem over $K$, for $i=1, \cdots, d+1$ :

$$
\int_{K} a\left(x, \frac{x}{\varepsilon}\right) \nabla w_{i}^{K} \nabla \varphi d x=\frac{1}{|K|} \int_{K} \varphi d x-\frac{1}{\left|e_{i}^{K}\right|} \int_{e_{i}^{K}} \varphi d s \quad \forall \varphi \in H^{1}(K),
$$

Equation (2.13) is the weak formulation of the following boundary value problem

$$
L_{\varepsilon} w_{i}^{K}=\frac{1}{|K|} \text { in } K, \quad-a\left(x, \frac{x}{\varepsilon}\right) \nabla w_{i}^{K} \cdot \nu^{K}=\left\{\begin{array}{cll}
\frac{1}{\left|e_{i}^{K}\right|} & \text { on } & e_{i}^{K} ; \\
0 & \text { on } & e_{j}^{K}, j \neq i .
\end{array}\right.
$$

Now let $\mathbf{p}_{i}^{K}=-a(x, x / \varepsilon) \nabla w_{i}^{K}$ and denote by $\operatorname{MS}(K)$ the multiscale finite element space spanned by $\mathbf{p}_{i}^{K}, i=1, \cdots, d+1$. Recall that $\operatorname{div} R_{i}^{K}=\frac{1}{|K|}$. We have

$$
\operatorname{div} \mathbf{p}_{i}^{K}=\operatorname{div} R_{i}^{K} \text { in } K, \text { and } \mathbf{p}_{i}^{K} \cdot \boldsymbol{\nu}^{K}=R_{i}^{K} \cdot \boldsymbol{\nu}^{K} \text { on } \partial K .
$$

Moreover, for any $\mathbf{q}_{h} \in \operatorname{MS}(K)$, the relations

$$
\int_{e_{i}^{K}} \mathbf{q}_{h} \cdot \boldsymbol{\nu}^{K} d s=0, \quad i=1, \cdots, d+1,
$$

imply $\mathbf{q}_{h}=0$ in $K$. The degrees of freedom for $\mathbf{q}_{h} \in \operatorname{MS}(K)$ can be chosen as the zeroth order moments of $\mathbf{q}_{h} \cdot \boldsymbol{\nu}^{K}$ on the sides or faces of $K$. In practical applications, the base functions $\mathbf{p}_{i}^{K}$ of $\operatorname{MS}(K)$ will be approximated by solving (2.13) on a triangulation of $K$ with a mesh size resolving $\varepsilon$ using the lowest-order Raviart-Thomas mixed finite element method.

Let $\Sigma=H(\operatorname{div} ; \Omega), Q=H_{0}(\operatorname{div} ; \Omega)$, and $M=L_{0}^{2}(\Omega)$. We define $\Sigma_{h}$ as the following multiscale finite element space for approximating the flux $\mathbf{p}_{\varepsilon}$ :

$$
\Sigma_{h}=\left\{\mathbf{q}_{h} \in H(\operatorname{div} ; \Omega):\left.\mathbf{q}_{h}\right|_{K} \in \operatorname{MS}(K), \forall K \in \mathcal{T}_{h}\right\} .
$$

Let $Q_{h}=\Sigma_{h} \cap Q$. To introduce an approximation of the boundary data $g$, we let $\mathcal{B}_{h}$ be the collection of all sides or faces of the triangulation $\mathcal{T}_{h}$ which lie on the boundary $\partial \Omega$. For any $e \in \mathcal{B}_{h}$ such that $e=\partial K \cap \partial \Omega$, we let $\mathbf{p}_{e} \in \operatorname{MS}(K)$ be the 
corresponding multiscale basis and define $g_{h}=\mathbf{q}_{0, h} \cdot \mathbf{n}$ on $\partial \Omega$ with $\mathbf{q}_{0, h} \in \Sigma_{h}$ given by

$$
\mathbf{q}_{0, h}=\sum_{e \in \mathcal{B}_{h}}\left(\int_{e} g d s\right) \mathbf{p}_{e} .
$$

We can now introduce the following discretization of (2.8)-2.9): find a pair $\left(\mathbf{p}_{h}, u_{h}\right)$ $\in \Sigma_{h} \times M_{h}$ such that $\mathbf{p}_{h} \cdot \mathbf{n}=g_{h}$ on $\partial \Omega$ and

$$
\begin{aligned}
& \left(\operatorname{div} \mathbf{p}_{h}, v_{h}\right)=\left(f, v_{h}\right) \quad \forall v_{h} \in M_{h}, \\
& \left(a^{-1}(x, x / \varepsilon) \mathbf{p}_{h}, \mathbf{q}_{h}\right)-\left(u_{h}, \operatorname{div} \mathbf{q}_{h}\right)=0 \quad \forall \mathbf{q}_{h} \in Q_{h} .
\end{aligned}
$$

We have the following theorem concerning the convergence of this discrete problem.

Theorem 2.1. The discrete problem (2.14)-(2.15) has a unique solution $\left(\mathbf{p}_{h}, u_{h}\right) \in$ $\Sigma_{h} \times M_{h}$ such that $\mathbf{p}_{h} \cdot \mathbf{n}=g_{h}$ on $\partial \Omega$. Moreover, if the homogenized solution $u_{0} \in H^{2}(\Omega) \cap W^{1, \infty}(\Omega)$ and the assumptions (H1)-(H2) are satisfied, then there exists a constant $C>0$, independent of $h$ and $\varepsilon$, such that

$$
\begin{aligned}
& \left\|\mathbf{p}_{\varepsilon}-\mathbf{p}_{h}\right\|_{\operatorname{div}, \Omega}+\left\|u_{\varepsilon}-u_{h}\right\|_{0, \Omega} \\
\leq & C(h+\varepsilon)\left(\left\|u_{0}\right\|_{2, \Omega}+\|f\|_{1, \Omega}+\left\|\mathbf{q}_{0}\right\|_{\operatorname{div}, \Omega}\right)+C \sqrt{\frac{\varepsilon}{h}}\left\|u_{0}\right\|_{1, \infty, \Omega} .
\end{aligned}
$$

The proof of this theorem is simpler than that of Theorem 2.2 below for the oversampling mixed multiscale finite element method, and will be omitted. We remark that the error estimate (2.16) is uniform as $\varepsilon \rightarrow 0$, which is in strong contrast to the error estimate (2.12), which blows up as $\varepsilon \rightarrow 0$. We also observe that the error estimate (2.16) deteriorates when $\varepsilon$ is of the same order as the mesh size $h$. This is due to the boundary layer effect of the multiscale bases, that is, the mismatch of the oscillating structure of $\mathbf{p}_{\varepsilon}$ and the linear behavior of the finite element solution $\mathbf{p}_{h}$ on the boundaries $\partial K, K \in \mathcal{T}_{h}$. This phenomenon is called resonant error in [18. The resonant error can be significantly reduced by an over-sampling technique introduced in [18]. The over-sampling technique is based on the observation that the width of the boundary layer is of order $O(\varepsilon)$. Thus, if one first constructs some intermediate base functions on a larger sample domain (with size larger than $(h+O(\epsilon)))$ and uses only the interior information from these intermediate base functions to construct the actual computational bases, then the boundary layer effect can be substantially reduced, resulting in more accurate approximations.

2.3. Over-sampling technique. In this subsection we will use the over-sampling technique [18 to construct new multiscale finite element bases for which the boundary layer effect of the bases $\mathbf{p}_{i}^{K}, i=1, \cdots, d+1$, is reduced.

For any $K \in \mathcal{T}_{h}$, we denote by $S=S(K)$ a macro-element which contains $K$ and satisfies following condition:

(H3): $h_{S} \leq C_{0} h_{K}$ and $\operatorname{dist}(\partial K, \partial S) \geq \delta_{0} h_{K}$ for some positive constants $C_{0}, \delta_{0}$ independent of $h$. The minimum angle of $S(K)$ is bounded below by some positive constant $\theta_{0}$ independent of $h$.

Let $\left\{R_{i}^{S}\right\}_{i=1}^{d+1}$ be the basis of $R T_{0}(S)$ which satisfies

$$
R_{i}^{S} \cdot \boldsymbol{\nu}^{S}=\left\{\begin{array}{cll}
\frac{1}{\left|e_{i}^{S}\right|} & \text { on } & e_{i}^{S}, \\
0 & \text { on } & e_{j}^{S}, j \neq i .
\end{array}\right.
$$


Recall that $V(S)$ stands for the subspace of $H^{1}(S)$ whose functions have zero average over $S$. Let $\left\{\mathbf{p}_{i}^{S}\right\}_{i=1}^{d+1}$ be the multiscale finite element basis of $\operatorname{MS}(S)$ defined as in (2.13), i.e., $\mathbf{p}_{i}^{S}=-a(x, x / \varepsilon) \nabla w_{i}^{S}$, where $w_{i}^{S} \in V(S)$ is the solution of the following problem over $S, i=1, \cdots, d+1$ :

$$
\int_{S} a\left(x, \frac{x}{\varepsilon}\right) \nabla w_{i}^{S} \nabla \varphi d x=\frac{1}{|S|} \int_{S} \varphi d x-\frac{1}{\left|e_{i}^{S}\right|} \int_{e_{i}^{S}} \varphi d s \quad \forall \varphi \in H^{1}(S) .
$$

Then we define the over-sampling finite element basis over $K$ by

$$
\overline{\mathbf{p}}_{i}^{K}=\left.c_{i j}^{K} \mathbf{p}_{j}^{S}\right|_{K} \quad \text { in } K
$$

where the constants $c_{i j}^{K}$ are chosen such that

$$
R_{i}^{K}=\left.c_{i j}^{K} R_{j}^{S}\right|_{K}
$$

The existence of the constants $c_{i j}^{K}$ is guaranteed because $\left\{R_{j}^{S}\right\}_{j=1}^{d+1}$ also forms the basis of $R T_{0}(K)$. Since $\operatorname{div} \mathbf{p}_{j}^{S}=\operatorname{div} R_{j}^{S}=1 /|S|$, we know that

$$
\operatorname{div} \overline{\mathbf{p}}_{i}^{K}=\operatorname{div} R_{i}^{K} \text { in } K \text {. }
$$

Here again, in practical applications, the base functions $\mathbf{p}_{i}^{S}$ of $\operatorname{MS}(S)$ will be further approximated by solving (2.17) on a triangulation of $S$ with a mesh size resolving $\varepsilon$ using the lowest order Raviart-Thomas finite element method. When applying the multiscale finite element method to solve (1.1)-(1.2), these base functions can be precomputed initially to generate the coarse grid operator. So this will be only a small overhead of the overall computations. This is also a common practice in many upscaling methods [10], [11.

We define $X=\prod_{K \in \mathcal{T}_{h}} H(\operatorname{div} ; K)$ with norm $\|\mathbf{q}\|_{X}=\left(\sum_{K \in \mathcal{T}_{h}}\|\mathbf{q}\|_{\text {div }, K}^{2}\right)^{1 / 2}$. Let $\Lambda(K)=\operatorname{span}\left\{\overline{\mathbf{p}}_{i}^{K}\right\}_{i=1}^{d+1}$ and $\Pi_{K}: \Lambda(K) \rightarrow R T_{0}(K)$ be the projection

$$
\Pi_{K} \mathbf{q}=c_{i} R_{i}^{K} \quad \text { if } \quad \mathbf{q}=c_{i} \overline{\mathbf{p}}_{i}^{K} \in \Lambda(K) .
$$

Let $\bar{X}_{h}$ be the finite element space

$$
\bar{X}_{h}=\left\{\mathbf{q}_{h} \in X:\left.\mathbf{q}_{h}\right|_{K} \in \Lambda(K), \quad \forall K \in \mathcal{T}_{h}\right\}
$$

and define $\Pi_{h}: \bar{X}_{h} \rightarrow \prod_{K \in \mathcal{T}_{h}} R T_{0}(K)$ through the relation $\left.\Pi_{h} \mathbf{q}_{h}\right|_{K}=\Pi_{K} \mathbf{q}_{h}$ for any $K \in \mathcal{T}_{h}, \mathbf{q}_{h} \in \bar{X}_{h}$. The over-sampling mixed multiscale finite element space for approximating the flux $\mathbf{p}_{\varepsilon}=-a(x, x / \varepsilon) \nabla u_{\varepsilon}$ is then defined as

$$
X_{h}=\left\{\mathbf{q}_{h} \in \bar{X}_{h}: \Pi_{h} \mathbf{q}_{h} \in W_{h} \subset H(\operatorname{div} ; \Omega)\right\} .
$$

We remark that, in general, $X_{h} \not \subset \Sigma=H(\operatorname{div} ; \Omega)$. Here, we require $\Pi_{h} \mathbf{q}_{h} \in W_{h}$ to impose some intrinsic continuity of the zeroth order moment of the normal components of $\mathbf{q}_{h} \in X_{h}$ across the interelement boundaries of the triangulation $\mathcal{T}_{h}$. Finally, let $G_{h}$ be the closed subspace of $X_{h}$ defined by

$$
G_{h}=\left\{\mathbf{q}_{h} \in \bar{X}_{h}: \Pi_{h} \mathbf{q}_{h} \in W_{h} \cap H_{0}(\operatorname{div} ; \Omega)\right\} .
$$

The over-sampling mixed finite element approximation of (2.14)-2.15) can be formulated as follows: find a pair $\left(\mathbf{p}_{h}, u_{h}\right) \in X_{h} \times M_{h}$ such that $\mathbf{p}_{h} \cdot \mathbf{n}=\bar{g}_{h}$ on $\partial \Omega$ and

$$
\begin{aligned}
\sum_{K \in \mathcal{T}_{h}} \int_{K} a^{-1} \mathbf{p}_{h} \cdot \mathbf{q}_{h} d x-\sum_{K \in \mathcal{T}_{h}} \int_{K} \operatorname{div} \mathbf{q}_{h} \cdot u_{h} d x & =0 \quad \forall \mathbf{q}_{h} \in G_{h}, \\
\sum_{K \in \mathcal{T}_{h}} \int_{K} \operatorname{div} \mathbf{p}_{h} \cdot v_{h} d x & =\left(f, v_{h}\right) \quad \forall v_{h} \in M_{h} .
\end{aligned}
$$


Here $\bar{g}_{h}=\overline{\mathbf{q}}_{0, h} \cdot \mathbf{n}$ is the approximation of the boundary value with $\overline{\mathbf{q}}_{0, h} \in X_{h}$ given by

$$
\overline{\mathbf{q}}_{0, h}=\sum_{e \in \mathcal{B}_{h}}\left(\int_{e} g d s\right) \overline{\mathbf{p}}_{e}
$$

for any $e \in \mathcal{B}_{h}$ such that $e=\partial K \cap \partial \Omega$, and $\overline{\mathbf{p}}_{e} \in \Lambda(K)$ is the corresponding over-sampling multiscale basis.

We have the following theorem about the convergence of the over-sampling mixed multiscale finite element method. The assumption (H4) will be made precise in $\S 4$ see equation (4.8). This is an assumption on the maximum bound of a boundary corrector term (in the multiple scale expansion) in terms of the gradient of the homogenized solution. The validity of this assumption can be easily proved for the corresponding Dirichlet boundary value problem, due to the maximum principle.

Theorem 2.2. The discrete problem (2.21) $-(2.22)$ has a unique solution $\left(\mathbf{p}_{h}, u_{h}\right) \in$ $X_{h} \times M_{h}$ such that $\mathbf{p}_{h} \cdot \mathbf{n}=\bar{g}_{h}$ on $\partial \Omega$. Moreover, if the homogenized solution $u_{0} \in H^{2}(\Omega) \cap W^{1, \infty}(\Omega)$ and the assumptions (H1)-(H4) are satisfied, then there exist constants $\delta>0$ and $C>0$, independent of $h$ and $\varepsilon$, such that if $h_{K} \leq \delta, \varepsilon / h_{K} \leq \delta$ for all $K \in \mathcal{T}_{h}$, the following error estimate holds:

$$
\begin{aligned}
\| \mathbf{p}_{\varepsilon}- & \mathbf{p}_{h}\left\|_{X}+\right\| u_{\varepsilon}-u_{h} \|_{0, \Omega} \\
\leq & C(\varepsilon+h)\left(\left\|u_{0}\right\|_{2, \Omega}+\|f\|_{1, \Omega}+\left\|\mathbf{q}_{0}\right\|_{\operatorname{div}, \Omega}\right) \\
& +C\left(\frac{\varepsilon}{h}+\sqrt{\varepsilon}\right)\left(\left\|u_{0}\right\|_{1, \infty, \Omega}+\|f\|_{0, \Omega}+\left\|\mathbf{q}_{0}\right\|_{\operatorname{div}, \Omega}\right) .
\end{aligned}
$$

The proof of this theorem, which will be given in $\$ 4$ depends on an abstract formulation for nonconforming mixed finite element methods, the homogenization results for elliptic equations with locally periodic coefficients in $\$ 3$, and a technique of "freezing coefficients" to deal with the local periodicity of the coefficients. This new technique can also be used to extend the convergence analysis in [19, 13] for displacement multiscale finite element methods to elliptic equations with locally periodic coefficients. We remark that locally periodic coefficients model a wide class of microstructures which include, in particular, the layered structures. Previous numerical experiments in 13 and our numerical experiments indicate that the over-sampling technique leads to significant improvement over the direct mixed multiscale finite element method (2.14)-(2.15).

\section{Homogenization Results}

In this section we summarize some homogenization results for the Neumann problem in the form to be used in this paper.

Let $D \subseteq \Omega$ be a domain in $\mathbf{R}^{d}$ with Lipschitz boundary $\Gamma$. Given $f \in L^{2}(D)$ and $g \in L^{2}(\Gamma)$ which satisfy the compatibility condition

$$
\int_{D} f d x=\int_{\Gamma} g d s
$$

we consider the following Neumann problem with rapidly oscillating coefficients: find $w_{\varepsilon} \in V(D)$ such that

$$
\int_{D} a\left(x, \frac{x}{\varepsilon}\right) \nabla w_{\varepsilon} \nabla \varphi d x=\int_{D} f \varphi d x-\int_{\Gamma} g \varphi d s \quad \forall \varphi \in H^{1}(D) .
$$


It is well-known that this problem admits a unique solution in $V(D)$ due to the compatibility condition (3.1). Let $w_{0} \in V(D)$ be the unique solution of the homogenized problem

$$
\int_{D} a^{*}(x) \nabla w_{0} \nabla \varphi d x=\int_{D} f \varphi d x-\int_{\Gamma} g \varphi d s \quad \forall \varphi \in H^{1}(D),
$$

where $a^{*}(x)$ is given by 2.6 .

In the following we will assume that $w_{0} \in H^{2}(D) \cap W^{1, \infty}(D)$. This regularity is valid, for example, when $f \in L^{p}(\Omega), g=\mathbf{q}_{0} \cdot \mathbf{n}$ on $\Gamma$ for some $\mathbf{q}_{0} \in W^{1, p}(\Omega)$, where $p>2$, and $D$ is a smooth domain [15] or a convex polyhedral domain in $\mathbf{R}^{2}$ or $\mathbf{R}^{3}$ 17], [23].

Now we set

$$
w_{1}^{\varepsilon}(x)=w_{0}(x)-\varepsilon \chi^{k}\left(x, \frac{x}{\varepsilon}\right) \frac{\partial w_{0}}{\partial x_{k}},
$$

where $\chi^{k}(x, y)$ is the solution of the cell problem (2.7). Then by simple calculations we get

$$
\begin{aligned}
a_{i j}\left(x, \frac{x}{\varepsilon}\right) \frac{\partial w_{1}^{\varepsilon}}{\partial x_{j}} & =a_{i j}^{*}(x) \frac{\partial w_{0}}{\partial x_{j}}-G_{i}^{k}\left(x, \frac{x}{\varepsilon}\right) \frac{\partial w_{0}}{\partial x_{k}} \\
& -\varepsilon a_{i j}\left(x, \frac{x}{\varepsilon}\right) \frac{\partial \chi^{k}}{\partial x_{j}}\left(x, \frac{x}{\varepsilon}\right) \frac{\partial w_{0}}{\partial x_{k}} \\
& -\varepsilon a_{i j}\left(x, \frac{x}{\varepsilon}\right) \chi^{k}\left(x, \frac{x}{\varepsilon}\right) \frac{\partial^{2} w_{0}}{\partial x_{j} \partial x_{k}},
\end{aligned}
$$

where

$$
G_{i}^{k}(x, y)=a_{i k}^{*}(x)-a_{i j}(x, y)\left(\delta_{k j}-\frac{\partial \chi^{k}}{\partial y_{j}}(x, y)\right) .
$$

Due to (2.6) and (2.7) we know that $\int_{Y} G_{i}^{k}(x, y) d y=0$ and $\partial G_{i}^{k}(x, y) / \partial y_{i}=0$. Thus there exist skew-symmetric matrices $\alpha^{k}(x, y)=\left(\alpha_{i j}^{k}(x, y)\right)$ such that [20, p.6]

$$
G_{i}^{k}(x, y)=\frac{\partial}{\partial y_{j}}\left(\alpha_{i j}^{k}(x, y)\right), \quad \int_{Y} \alpha_{i j}^{k}(x, y) d y=0 .
$$

With this notation, we can rewrite

$$
\begin{aligned}
G_{i}^{k}\left(x, \frac{x}{\varepsilon}\right) \frac{\partial w_{0}}{\partial x_{k}}= & \varepsilon \frac{\partial}{\partial x_{j}}\left(\alpha_{i j}^{k}\left(x, \frac{x}{\varepsilon}\right) \frac{\partial w_{0}}{\partial x_{k}}\right)-\varepsilon \alpha_{i j}^{k}\left(x, \frac{x}{\varepsilon}\right) \frac{\partial^{2} w_{0}}{\partial x_{j} \partial x_{k}} \\
& -\varepsilon \frac{\partial \alpha_{i j}^{k}}{\partial x_{j}}\left(x, \frac{x}{\varepsilon}\right) \frac{\partial w_{0}}{\partial x_{k}}
\end{aligned}
$$

We define $\theta_{\varepsilon} \in V(D)$ as the solution of the following problem:

$$
\int_{D} a\left(x, \frac{x}{\varepsilon}\right) \nabla \theta_{\varepsilon} \nabla \varphi d x=\int_{D} \frac{\partial}{\partial x_{j}}\left(\alpha_{i j}^{k}\left(x, \frac{x}{\varepsilon}\right) \frac{\partial w_{0}}{\partial x_{k}}\right) \frac{\partial \varphi}{\partial x_{i}} d x \quad \forall \varphi \in H^{1}(D) .
$$

Note that $\alpha_{i j}^{k}=-\alpha_{j i}^{k}$. This problem can be regarded as the weak formulation of the elliptic problem

$$
L_{\varepsilon} \theta_{\varepsilon}=0 \quad \text { in } D, \quad a\left(x, \frac{x}{\varepsilon}\right) \nabla \theta_{\varepsilon} \cdot \nu^{D}=\frac{\partial}{\partial x_{j}}\left(\alpha_{i j}^{k}\left(x, \frac{x}{\varepsilon}\right) \frac{\partial w_{0}}{\partial x_{k}}\right) \cdot \nu_{i}^{D} \quad \text { on } \Gamma,
$$


where $\boldsymbol{\nu}^{D}=\left(\nu_{1}^{D}, \cdots, \nu_{d}^{D}\right)$ is the unit outer normal to $\Gamma$. Hence $\theta_{\varepsilon}$ plays the role of a boundary corrector in the asymptotic expansion for $w_{\varepsilon}$. The following theorem generalizes similar results in [20, §1.4] for periodic coefficients.

Theorem 3.1. Assume that $w_{0} \in H^{2}(D) \cap W^{1, \infty}(D)$. Then there exists a constant $C$, independent of $\varepsilon$, the domain $D$, and the data $f$ and $g$, such that

$$
\left\|\nabla w_{\varepsilon}-\nabla\left(w_{1}^{\varepsilon}+\varepsilon \theta_{\varepsilon}\right)\right\|_{0, D} \leq C \varepsilon\left(\left|w_{0}\right|_{2, D}+\left|w_{0}\right|_{1, D}\right) .
$$

Moreover, the boundary corrector $\theta_{\varepsilon}$ satisfies the estimate

$$
\left\|\varepsilon \nabla \theta_{\varepsilon}\right\|_{0, D} \leq C \varepsilon\left(\left|w_{0}\right|_{2, D}+\left|w_{0}\right|_{1, D}\right)+C \sqrt{\varepsilon|\Gamma|}\left|w_{0}\right|_{1, \infty, D},
$$

where $|\Gamma|$ stands for the length of the boundary $\Gamma$ if $d=2$, and the surface area of $\Gamma$ if $d=3$.

Proof. For the sake of completeness, we sketch the proof here in order to track down the exact dependence of the constant $C$ in (3.8) and (3.9). First, we deduce from (3.2), (3.3), (3.5)-(3.6), and (3.7) that

$$
\begin{aligned}
& \int_{D} a\left(x, \frac{x}{\varepsilon}\right) \nabla\left(w_{\varepsilon}-w_{1}^{\varepsilon}-\varepsilon \theta_{\varepsilon}\right) \nabla \varphi d x \\
= & \varepsilon \int_{D}\left[a_{i j}\left(x, \frac{x}{\varepsilon}\right) \chi^{k}\left(x, \frac{x}{\varepsilon}\right) \frac{\partial^{2} w_{0}}{\partial x_{j} \partial x_{k}}+a_{i j}\left(x, \frac{x}{\varepsilon}\right) \frac{\partial \chi^{k}}{\partial x_{j}}\left(x, \frac{x}{\varepsilon}\right) \frac{\partial w_{0}}{\partial x_{k}}\right] \frac{\partial \varphi}{\partial x_{i}} d x \\
- & \varepsilon \int_{D}\left[\alpha_{i j}^{k}\left(x, \frac{x}{\varepsilon}\right) \frac{\partial^{2} w_{0}}{\partial x_{j} \partial x_{k}}+\frac{\partial \alpha_{i j}^{k}}{\partial x_{j}}\left(x, \frac{x}{\varepsilon}\right) \frac{\partial w_{0}}{\partial x_{k}}\right] \frac{\partial \varphi}{\partial x_{i}} d x \quad \forall \varphi \in H^{1}(D) .
\end{aligned}
$$

This implies (3.8) after taking $\varphi=w_{\varepsilon}-w_{1}^{\varepsilon}-\varepsilon \theta_{\varepsilon} \in H^{1}(D)$ and using the ellipticity condition (2.3).

To show (3.9), we introduce a cutoff function $\zeta_{\varepsilon} \in C_{0}^{\infty}(D), 0 \leq \zeta_{\varepsilon} \leq 1$ in $D$, $\zeta_{\varepsilon}=1$ outside the $\varepsilon$-neighborhood of the boundary $\Gamma$, and $\left|\nabla \zeta_{\varepsilon}\right| \leq \widehat{C} / \varepsilon$ in $D$ with $\widehat{C}$ independent of $\varepsilon$ and $D$. Then, we have

$$
\begin{aligned}
\frac{\partial}{\partial x_{j}}\left(\alpha_{i j}^{k}\left(x, \frac{x}{\varepsilon}\right) \frac{\partial w_{0}}{\partial x_{k}}\right) & =\frac{\partial}{\partial x_{j}}\left(\alpha_{i j}^{k}\left(x, \frac{x}{\varepsilon}\right) \frac{\partial w_{0}}{\partial x_{k}} \zeta_{\varepsilon}\right) \\
& +\frac{\partial}{\partial x_{j}}\left(\alpha_{i j}^{k}\left(x, \frac{x}{\varepsilon}\right) \frac{\partial w_{0}}{\partial x_{k}}\left(1-\zeta_{\varepsilon}\right)\right) .
\end{aligned}
$$

Note that the first term is divergence free since the matrix $\alpha^{k}$ is skew-symmetric and with compact support. We have from (3.7) that

$$
\int_{D} a\left(x, \frac{x}{\varepsilon}\right) \nabla \theta_{\varepsilon} \nabla \varphi d x=\int_{D} \frac{\partial}{\partial x_{j}}\left(\alpha_{i j}^{k}\left(x, \frac{x}{\varepsilon}\right) \frac{\partial w_{0}}{\partial x_{k}}\left(1-\zeta_{\varepsilon}\right)\right) \frac{\partial \varphi}{\partial x_{i}} d x \quad \forall \varphi \in H^{1}(D) .
$$

Denote $D_{\varepsilon}=\operatorname{supp}\left(1-\zeta_{\varepsilon}\right)$; then $\left|D_{\varepsilon}\right| \leq \varepsilon|\Gamma|$ and thus

$$
\begin{aligned}
& \varepsilon\left\|\frac{\partial}{\partial x_{j}}\left(\alpha_{i j}^{k}\left(\cdot, \frac{\dot{\varepsilon}}{\varepsilon}\right) \frac{\partial w_{0}}{\partial x_{k}}\left(1-\zeta_{\varepsilon}\right)\right)\right\|_{0, D} \\
& \leq \varepsilon\left\|\frac{\partial \alpha_{i j}^{k}}{\partial x_{j}}\left(\cdot, \frac{\cdot}{\varepsilon}\right) \frac{\partial w_{0}}{\partial x_{k}}\left(1-\zeta_{\varepsilon}\right)\right\|_{0, D}+\varepsilon\left\|\alpha_{i j}^{k}\left(\cdot, \frac{\cdot}{\varepsilon}\right) \frac{\partial^{2} w_{0}}{\partial x_{j} \partial x_{k}}\left(1-\zeta_{\varepsilon}\right)\right\|_{0, D} \\
& +\left\|\left(\frac{\partial}{\partial y_{j}} \alpha_{i j}^{k}\right)\left(\cdot, \frac{\dot{ }}{\varepsilon}\right) \frac{\partial w_{0}}{\partial x_{k}}\left(1-\zeta_{\varepsilon}\right)\right\|_{0, D_{\varepsilon}}+\varepsilon\left\|\alpha_{i j}^{k}\left(\cdot, \frac{\dot{ }}{\varepsilon}\right) \frac{\partial w_{0}}{\partial x_{k}} \frac{\partial \zeta_{\varepsilon}}{\partial x_{j}}\right\|_{0, D_{\varepsilon}} \\
& \leq C \varepsilon\left(\left|w_{0}\right|_{2, D}+\left|w_{0}\right|_{1, D}\right)+C \sqrt{\varepsilon|\Gamma|}\left|w_{0}\right|_{1, \infty, D} .
\end{aligned}
$$

This completes the proof. 
An important open question is to show that the boundary corrector $\theta_{\varepsilon}$ is of first order, i.e., $\left\|\varepsilon \theta_{\varepsilon}\right\|_{0, \infty, D} \leq C \varepsilon\left|w_{0}\right|_{1, \infty, D}$ for some constant $C$ independent of $\varepsilon$. Similar results for the boundary corrector for Dirichlet problem are known (cf., e.g., [4], [2]).

\section{Convergence analysis}

The purpose of this section is to prove Theorem [2.2. We first recall the abstract formulation for the nonconforming mixed finite element method in $\S 4.1$, then establish the discrete inf-sup condition in $\S 4.2$, and finally prove the error estimate in $\S 4.3$.

4.1. Abstract results. The abstract results in this section modify slightly the standard Babuška-Brezzi theory [5], [16] in that we take into account the discretization of inhomogeneous boundary conditions. Another way to deal with the approximation of boundary conditions is considered in [7, which, however, is not applicable here due to the oscillatory nature of the coefficient in (2.8)-(2.9) . Let $\Sigma$ and $M$ be two Hilbert spaces with norms $\|\cdot\|_{\Sigma}$ and $\|\cdot\|_{M}$ respectively. Denote by $\Sigma^{\prime}$ and $M^{\prime}$ the corresponding dual spaces, and by $\langle\cdot, \cdot\rangle_{\Sigma^{\prime} \times \Sigma}$ and $\langle\cdot, \cdot\rangle_{M^{\prime} \times M}$ the duality pairings. Let $a: \Sigma \times \Sigma \rightarrow \mathbf{R}$ and $b: \Sigma \times M \rightarrow \mathbf{R}$ be two continuous bilinear forms. Let $Q$ be a closed subspace of $\Sigma$. Given $p_{0} \in \Sigma, l \in \Sigma^{\prime}$, and $\rho \in M^{\prime}$, we consider the following variational problem: find a pair $(p, \lambda) \in \Sigma \times M$ such that $p-p_{0} \in Q$ and

$$
\begin{aligned}
a(p, q)+b(q, \lambda) & =\langle l, q\rangle_{\Sigma^{\prime} \times \Sigma} \quad \forall q \in Q, \\
b(p, \mu) & =\langle\rho, \mu\rangle_{M^{\prime} \times M} \quad \forall \mu \in M .
\end{aligned}
$$

Define $V=\{q \in Q: b(q, \mu)=0, \quad \forall \mu \in M\}$. The following result concerning the existence and uniqueness of solutions of the abstract problem (4.1)-(4.2) can be easily proved by rewriting (4.1)-(4.2) in terms of $\tilde{p}=p-p_{0} \in Q$ and using the standard theory in [5, §II.1], [16, §I.4].

Lemma 4.1. Assume that the bilinear form $a(\cdot, \cdot)$ is $V$-elliptic, i.e., there exists a constant $\alpha>0$ such that

$$
a(q, q) \geq \alpha\|q\|_{\Sigma}^{2} \quad \forall q \in V,
$$

and the bilinear form $b(\cdot, \cdot)$ satisfies the following inf-sup condition for some constant $\beta>0$ :

$$
\inf _{0 \neq \mu \in M} \sup _{0 \neq q \in Q} \frac{b(q, \mu)}{\|q\|_{\Sigma}\|\mu\|_{M}} \geq \beta .
$$

Then, the abstract problem (4.1)-(4.2) has a unique solution $(p, \lambda) \in \Sigma \times M$ such that $p-p_{0} \in Q$.

We consider now nonconforming approximations of the mixed problem (4.1)(4.2). Let $X$ be a Hilbert space with the norm $\|\cdot\|_{X}$ such that $\Sigma \subset X$. We denote by $a_{h}: X \times X \rightarrow \mathbf{R}$ and $b_{h}: X \times M \rightarrow \mathbf{R}$ continuous bilinear forms which are approximations of the bilinear forms $a: \Sigma \times \Sigma \rightarrow \mathbf{R}$ and $b: \Sigma \times M \rightarrow \mathbf{R}$, respectively. The index $h$ will eventually refer to the mesh for which these approximations are derived. Furthermore, we assume that $l \in X^{\prime}$, where $X^{\prime}$ is the dual space of $X$. Let $X_{h} \subset X$ and $M_{h} \subset M$ be finite dimensional spaces. Denote by $G_{h}$ a closed subspace of $X_{h}$. Let $p_{0, h} \in X_{h}$ be an approximation of $p_{0} \in \Sigma$. We consider the 
following approximation of (4.1)-(4.2): find a pair $\left(p_{h}, \lambda_{h}\right) \in X_{h} \times M_{h}$ such that $p_{h}-p_{0, h} \in G_{h}$ and

$$
\begin{aligned}
a_{h}\left(p_{h}, q_{h}\right)+b_{h}\left(q_{h}, \lambda_{h}\right) & =\left\langle l, q_{h}\right\rangle_{X^{\prime} \times X} \quad \forall q_{h} \in G_{h}, \\
b_{h}\left(p_{h}, \mu_{h}\right) & =\left\langle\rho, \mu_{h}\right\rangle_{M^{\prime} \times M} \quad \forall \mu_{h} \in M_{h} .
\end{aligned}
$$

Note that we have not assumed $X_{h} \subset \Sigma$, so (4.3)-(4.4) is a nonconforming discretization of the variational problem (4.1)-(4.2). Let $Z_{h}=\left\{q_{h} \in G_{h}: b_{h}\left(q_{h}, \mu_{h}\right)=\right.$ $\left.0, \forall \mu_{h} \in M_{h}\right\}$. We have the following abstract result, which accounts for the inhomogeneous boundary conditions and can be proved by modifying the argument in [5, §II.2.6].

Lemma 4.2. Assume that $a_{h}(\cdot, \cdot)$ is uniformly coercive in $Z_{h}$, i.e., there exists a constant $\alpha^{*}>0$, independent of $h$, such that

$$
a_{h}\left(q_{h}, q_{h}\right) \geq \alpha^{*}\left\|q_{h}\right\|_{X}^{2} \quad \forall q_{h} \in Z_{h},
$$

and $b_{h}(\cdot, \cdot)$ satisfies the following discrete inf-sup condition for some constant $\beta^{*}>$ 0 independent of $h$ :

$$
\inf _{0 \neq \mu_{h} \in M_{h}} \sup _{0 \neq q_{h} \in G_{h}} \frac{b_{h}\left(q_{h}, \mu_{h}\right)}{\left\|q_{h}\right\|_{X}\left\|\mu_{h}\right\|_{M}} \geq \beta^{*} .
$$

Then, the discrete problem (4.3)-(4.4) has a unique solution $\left(p_{h}, \lambda_{h}\right) \in X_{h} \times M_{h}$ such that $p_{h}-p_{0, h} \in G_{h}$. Moreover, the solution $\left(p_{h}, \lambda_{h}\right)$ fulfills the following error estimate:

$$
\begin{aligned}
\| p- & p_{h}\left\|_{X}+\right\| \lambda-\lambda_{h} \|_{M} \\
\leq & C\left(\inf _{\sigma_{h} \in X_{h}, \sigma_{h}-p_{0, h} \in G_{h}}\left\|p-\sigma_{h}\right\|_{X}+\inf _{\mu_{h} \in M_{h}}\left\|\lambda-\mu_{h}\right\|_{M}\right) \\
& +C \sup _{0 \neq q_{h} \in G_{h}} \frac{\left\langle l, q_{h}\right\rangle_{X^{\prime} \times X}-a_{h}\left(p, q_{h}\right)-b_{h}\left(q_{h}, \lambda\right)}{\left\|q_{h}\right\|_{X}},
\end{aligned}
$$

where the constant $C>0$ depends only on $\alpha^{*}, \beta^{*}$ and the operator norms $\left\|a_{h}\right\|$, $\left\|b_{h}\right\|$.

The last term at the right-hand side of the above abstract error estimate, which vanishes if $X_{h} \subset \Sigma$, represents the nonconforming error.

4.2. The discrete inf-sup condition. We will use the abstract framework in $\S 4.1$ to study the discrete problem (2.21)-(2.22). Thus we define $X=\prod_{K \in \mathcal{T}_{h}} H(\operatorname{div} ; K)$ with the norm $\|\mathbf{q}\|_{X}=\left(\sum_{K \in \mathcal{T}_{h}}\|\mathbf{q}\|_{\text {div }, K}^{2}\right)^{1 / 2}$, and introduce the bilinear forms $a_{h}: X \times X \rightarrow \mathbf{R}$ and $b_{h}: X \times L^{2}(\Omega) \rightarrow \mathbf{R}$ as

$$
\begin{aligned}
& a_{h}(\mathbf{p}, \mathbf{q})=\sum_{K \in \mathcal{T}_{h}} \int_{K} a^{-1}\left(x, \frac{x}{\varepsilon}\right) \mathbf{p} \cdot \mathbf{q} d x \quad \forall \mathbf{p}, \mathbf{q} \in X, \\
& b_{h}(\mathbf{q}, v)=-\sum_{K \in \mathcal{T}_{h}} \int_{K} \operatorname{div} \mathbf{q} \cdot v d x \quad \forall \mathbf{q} \in X, v \in L^{2}(\Omega) .
\end{aligned}
$$

It is clear that the operator norms satisfy $\left\|a_{h}\right\| \leq \gamma^{-1}$ and $\left\|b_{h}\right\| \leq 1$, where $\gamma$ is the constant in (2.3).

The over-sampling mixed finite element approximation (2.21)-(2.22) is then equivalent to the following form: find a pair $\left(\mathbf{p}_{h}, u_{h}\right) \in X_{h} \times M_{h}$ such that $\mathbf{p}_{h} \cdot \mathbf{n}=\bar{g}_{h}$ 
on $\partial \Omega$ and

$$
\begin{aligned}
a_{h}\left(\mathbf{p}_{h}, \mathbf{q}_{h}\right)+b_{h}\left(\mathbf{q}_{h}, u_{h}\right) & =0 \quad \forall \mathbf{q}_{h} \in G_{h}, \\
b_{h}\left(\mathbf{p}_{h}, v_{h}\right) & =-\left(f, v_{h}\right) \quad \forall v_{h} \in M_{h} .
\end{aligned}
$$

To study the properties of this discrete problem, we need the following lemma, which is crucial to the derivation of the discrete inf-sup condition for the bilinear form $b_{h}$.

Lemma 4.3. There exists a linear operator $\tau_{h}: W_{h} \rightarrow X_{h}$ such that for any $\mathbf{q} \in W_{h}$ and $K \in \mathcal{T}_{h}$ we have $\operatorname{div} \tau_{h} \mathbf{q}=\operatorname{div} \mathbf{q}$ in $K$ and $\left\|\tau_{h} \mathbf{q}\right\|_{\operatorname{div}, K} \leq C\|\mathbf{q}\|_{\operatorname{div}, K}$ for some constant $C$ independent of $h$.

Proof. For any $\mathbf{q} \in W_{h}$ and $K \in \mathcal{T}_{h}$, we have $\left.\mathbf{q}\right|_{K}=\beta_{i}^{K} R_{i}^{K} \in R T_{0}(K)$ for some constants $\beta_{i}^{K}, i=1, \cdots, d+1$. We are going to define the desired operator $\tau_{h}$ through the relation $\left.\tau_{h}\right|_{K}=\tau_{K}$ for any $K \in \mathcal{T}_{h}$ with the local form of the operator $\tau_{K}$ defined by

$$
\tau_{K} \mathbf{q}=\beta_{i}^{K} \overline{\mathbf{p}}_{i}^{K} .
$$

This implies that $\Pi_{K}\left(\tau_{K} \mathbf{q}\right)=\mathbf{q}$ in $K$. Thus, $\Pi_{h}\left(\tau_{h} \mathbf{q}\right)=\mathbf{q} \in W_{h}$, i.e., the operator $\tau_{h}: W_{h} \rightarrow X_{h}$ is well-defined. Moreover, by (2.20), we have $\operatorname{div} \tau_{h} \mathbf{q}=\operatorname{div} \mathbf{q}$ in $K$.

It remains to show the stability estimate in this lemma. To do so, we let $\bar{w}_{\varepsilon}^{S}=\beta_{i}^{K} c_{i j}^{K} w_{j}^{S}$, where $w_{j}^{S}$ is defined in (2.17); then we have $\tau_{K} \mathbf{q}=\left.\beta_{i}^{K} c_{i j}^{K} \mathbf{p}_{j}^{S}\right|_{K}=$ $-a(x, x / \varepsilon) \nabla \bar{w}_{\varepsilon}^{S}$. Define $\hat{\mathbf{q}}=\beta_{i}^{K} c_{i j}^{K} R_{j}^{S}$ and $\widehat{\tau_{K} \mathbf{q}}=\beta_{i}^{K} c_{i j}^{K} \mathbf{p}_{j}^{S}$ in $S$. Then from the definition of $\mathbf{p}_{j}^{S}$, we have $\operatorname{div} \hat{\mathbf{q}}=\operatorname{div} \widehat{\tau_{K} \mathbf{q}}$ in $S$ and $\hat{\mathbf{q}} \cdot \boldsymbol{\nu}^{S}=\widehat{\tau_{K} \mathbf{q}} \cdot \boldsymbol{\nu}^{S}$ on $\partial S$. Now, for any $\varphi \in H^{1}(S)$, we have, after integration by parts,

$$
\begin{aligned}
\int_{S} a\left(x, \frac{x}{\varepsilon}\right) \nabla \bar{w}_{\varepsilon}^{S} \nabla \varphi d x & =-\int_{S} \widehat{\tau_{K} \mathbf{q}} \cdot \nabla \varphi d x \\
& =\int_{S} \operatorname{div} \widehat{\tau_{K} \mathbf{q}} \cdot \varphi d x-\int_{\partial S}\left(\widehat{\tau_{K} \mathbf{q}} \cdot \boldsymbol{\nu}^{S}\right) \varphi d x \\
& =\int_{S} \operatorname{div} \hat{\mathbf{q}} \cdot \varphi d x-\int_{\partial S}\left(\hat{\mathbf{q}} \cdot \boldsymbol{\nu}^{S}\right) \varphi d x \\
& =-\int_{S} \hat{\mathbf{q}} \cdot \nabla \varphi d x .
\end{aligned}
$$

By taking $\varphi=\bar{w}_{\varepsilon}^{S}$ in above equality, we get $\left\|\widehat{\tau_{K} \mathbf{q}}\right\|_{0, S} \leq C\|\hat{\mathbf{q}}\|_{0, S}$. To conclude the proof we only need to notice that

$$
\|\hat{\mathbf{q}}\|_{0, S} \leq C\|\hat{\mathbf{q}}\|_{0, K}=C\|\mathbf{q}\|_{0, K}
$$

by the assumption (H3) and (2.19). This completes the proof.

Lemma 4.4. The discrete problem (4.5)-(4.6) has a unique solution $\left(\mathbf{p}_{h}, u_{h}\right) \in$ $X_{h} \times M_{h}$ such that $\mathbf{p}_{h} \cdot \mathbf{n}-\bar{g}_{h} \in G_{h}$. Moreover, the following error estimate holds:

$$
\begin{aligned}
& \left\|\mathbf{p}_{\varepsilon}-\mathbf{p}_{h}\right\|_{X}+\left\|u_{\varepsilon}-u_{h}\right\|_{0, \Omega} \\
& \leq C\left(\inf _{v_{h} \in M_{h}}\left\|u_{\varepsilon}-v_{h}\right\|_{0, \Omega}+\inf _{\mathbf{q}_{h} \in X_{h}, \mathbf{q}_{h} \cdot \mathbf{n}-\bar{g}_{h} \in G_{h}}\left\|\mathbf{p}_{\varepsilon}-\mathbf{q}_{h}\right\|_{X}\right) \\
& \quad+C \sup _{0 \neq \mathbf{q}_{h} \in G_{h}} \frac{\left|a_{h}\left(\mathbf{p}_{\varepsilon}, \mathbf{q}_{h}\right)+b_{h}\left(\mathbf{q}_{h}, u_{\varepsilon}\right)\right|}{\left\|\mathbf{q}_{h}\right\|_{X}}
\end{aligned}
$$


Proof. We apply the abstract Lemma 4.2 First, we note that, for any $K \in \mathcal{T}_{h}$ and $\mathbf{q}_{h} \in G_{h}$, by (2.20), $\operatorname{div} \mathbf{q}_{h}=\operatorname{div} \Pi_{h} \mathbf{q}_{h}$ in $K$. Therefore, $b_{h}\left(\mathbf{q}_{h}, 1\right)=b_{h}\left(\Pi_{h} \mathbf{q}_{h}, 1\right)=$ 0 since $\Pi_{h} \mathbf{q}_{h} \cdot \mathbf{n}=0$ on $\partial \Omega$ for any $\mathbf{q}_{h} \in G_{h}$. Now, since div $\mathbf{q}_{h}$ is constant over $K$, we have

$$
\begin{aligned}
Z_{h} & =\left\{\mathbf{q}_{h} \in G_{h}: b_{h}\left(\mathbf{q}_{h}, v_{h}\right)=0 \quad \forall v_{h} \in M_{h}\right\} \\
& =\left\{\mathbf{q}_{h} \in G_{h}: \operatorname{div} \mathbf{q}_{h}=0\right\}
\end{aligned}
$$

which implies clearly the $Z_{h}$ ellipticity of the bilinear form $a_{h}(\cdot, \cdot)$ after using (2.3).

Next, we check the discrete inf-sup condition. It follows from (2.10)-(2.11) that the discrete inf-sup condition is true for the Raviart-Thomas element [27], [5]:

$$
\inf _{0 \neq v_{h} \in M_{h}} \sup _{0 \neq \mathbf{q}_{h} \in W_{h}^{0}} \frac{b\left(\mathbf{q}_{h}, v_{h}\right)}{\left\|\mathbf{q}_{h}\right\|_{\operatorname{div}, \Omega}\left\|v_{h}\right\|_{0, \Omega}} \geq C_{1},
$$

where $W_{h}^{0}=W_{h} \cap H_{0}(\operatorname{div} ; \Omega)$ and $C_{1}>0$ is a constant independent of $h$. We now use Lemma 4.3 to conclude that, for any $v_{h} \in M_{h}$,

$$
\begin{aligned}
\sup _{0 \neq \mathbf{q}_{h} \in G_{h}} \frac{b_{h}\left(\mathbf{q}_{h}, v_{h}\right)}{\left\|\mathbf{q}_{h}\right\|_{X}} & \geq \sup _{0 \neq \mathbf{q} \in W_{h}^{0}} \frac{b_{h}\left(\tau_{h} \mathbf{q}, v_{h}\right)}{\left\|\tau_{h} \mathbf{q}\right\|_{X}} \\
& \geq \frac{1}{C} \sup _{0 \neq \mathbf{q} \in W_{h}^{0}} \frac{b_{h}\left(\mathbf{q}, v_{h}\right)}{\|\mathbf{q}\|_{\operatorname{div}, \Omega}} \\
& =\frac{1}{C} \sup _{0 \neq \mathbf{q} \in W_{h}^{0}} \frac{b\left(\mathbf{q}, v_{h}\right)}{\|\mathbf{q}\|_{\operatorname{div}, \Omega}} \geq \frac{C_{1}}{C}\left\|v_{h}\right\|_{0, \Omega} .
\end{aligned}
$$

This completes the proof.

4.3. Proof of Theorem [2.2. The error analysis in this subsection depends crucially on the behavior of the boundary corrector in the homogenization theory. Let $w_{\varepsilon} \in V(D)$ and $w_{0} \in V(D)$ be the respective solutions of problems (3.2) and (3.3) in $\S$. Let $\theta_{\varepsilon} \in V(D)$ be the boundary corrector defined in (3.7). We make the following assumption on the behavior of $\theta_{\varepsilon}$ :

(H4) There exists a constant $C$, independent of $\varepsilon$ and $D$, such that the following estimate is valid:

$$
\left\|\theta_{\varepsilon}\right\|_{0, \infty, D} \leq C\left\|\nabla w_{0}\right\|_{0, \infty, D} .
$$

This assumption, which is evident from our numerical experiments, is the basis of the over-sampling technique to reduce the resonant error. We notice that here $\theta_{\varepsilon}$ is the boundary corrector for the homogenization problem with Neumann boundary conditions. Such an estimate has been obtained for the homogenization problem with Dirichlet boundary conditions, for which the maximum principle can be used. However, this estimate is yet to be proved for the homogenization problem with Neumann boundary conditions.

Now for any subdomain $D^{\prime} \subset \bar{D}^{\prime} \subset D$ with $R=\operatorname{dist}\left(D^{\prime}, \partial D\right)>0$, the interior estimate in [2] yields

$$
\left\|\nabla \theta_{\varepsilon}\right\|_{0, D^{\prime}} \leq C R^{-1}\left\|\theta_{\varepsilon}\right\|_{0, D} .
$$

Thus, the assumption (H4) implies that there exists a constant $C$, independent of $\varepsilon, R$, and the domains $D^{\prime}$ and $D$, such that

$$
\left\|\nabla \theta_{\varepsilon}\right\|_{0, D^{\prime}} \leq C R^{-1}|D|^{1 / 2}\left\|\nabla w_{0}\right\|_{0, \infty, D} .
$$


The starting point of our error analysis is the error estimate in Lemma 4.4. We are going to estimate the three terms on the right-hand side of (4.7) in order.

Lemma 4.5. There exists a constant $C>0$, independent of $h$ and $\varepsilon$, such that

$$
\inf _{v_{h} \in M_{h}}\left\|u_{\varepsilon}-v_{h}\right\|_{0, \Omega} \leq C h\left(\|f\|_{0, \Omega}+\left\|\mathbf{q}_{0}\right\|_{\operatorname{div}, \Omega}\right) .
$$

Proof. It is easy to see from (2.1)-(2.2) that

$$
\left\|\nabla u_{\varepsilon}\right\|_{0, \Omega}+\left\|\mathbf{p}_{\varepsilon}\right\|_{0, \Omega} \leq C\left(\|f\|_{0, \Omega}+\left\|\mathbf{q}_{0}\right\|_{\text {div }, \Omega}\right) .
$$

For $u_{\varepsilon} \in V(\Omega)$, let us define a function

$$
\bar{u}_{h}=\frac{1}{|K|} \int_{K} u_{\varepsilon} d x \quad \text { in } K,
$$

whose average over $\Omega$ vanishes because $u_{\varepsilon} \in L_{0}^{2}(\Omega)$, and thus $\bar{u}_{h} \in M_{h}$. Now standard finite element interpolation estimates imply that

$$
\begin{aligned}
\inf _{v_{h} \in M_{h}}\left\|u_{\varepsilon}-v_{h}\right\|_{0, \Omega} \leq\left\|u_{\varepsilon}-\bar{u}_{h}\right\|_{0, \Omega} & \leq C h\left\|\nabla u_{\varepsilon}\right\|_{0, \Omega} \\
& \leq C h\left(\|f\|_{0, \Omega}+\left\|\mathbf{q}_{0}\right\|_{\operatorname{div}, \Omega}\right) .
\end{aligned}
$$

This completes the proof.

Lemma 4.6. Under the assumptions ( $\mathrm{H} 1)-(\mathrm{H} 4)$ there exists a constant $C>0$, independent of $h$ and $\varepsilon$, such that

$$
\begin{aligned}
\inf _{\mathbf{q}_{h} \in X_{h}, \mathbf{q}_{h} \cdot \mathbf{n}-\bar{g}_{h} \in G_{h}}\left\|\mathbf{p}_{\varepsilon}-\mathbf{q}_{h}\right\|_{X} & \leq C(\varepsilon+h)\left(\left\|u_{0}\right\|_{2, \Omega}+\|f\|_{1, \Omega}\right) \\
& +C\left(\frac{\varepsilon}{h}+\sqrt{\varepsilon}\right)\left\|u_{0}\right\|_{1, \infty, \Omega} .
\end{aligned}
$$

To prove this lemma, we let $\mathbf{p}_{0}=-a^{*}(x) \nabla u_{0} \in H(\operatorname{div} ; \Omega)$ and define $\mathbf{t}_{h} \in \bar{X}_{h}$ on each $K \in \mathcal{T}_{h}$ as

$$
\left.\mathbf{t}_{h}\right|_{K}=\gamma_{i}^{K} \overline{\mathbf{p}}_{i}^{K} \quad \text { with } \quad \gamma_{i}^{K}=\int_{e_{i}^{K}} \mathbf{p}_{0} \cdot \boldsymbol{\nu}^{K} d s .
$$

By the definition of $R_{i}^{K}$ we know that $\left.\Pi_{h} \mathbf{t}_{h}\right|_{K}=\gamma_{i}^{K} R_{i}^{K}=r_{K} \mathbf{p}_{0}=\left.r_{h} \mathbf{p}_{0}\right|_{K}$, where the operator $r_{h}: H(\operatorname{div} ; \Omega) \cap H^{1}(\Omega)^{d} \rightarrow W_{h}$ is defined in $\rrbracket$. Thus, $\Pi_{h} \mathbf{t}_{h} \in W_{h}$, which implies $\mathbf{t}_{h} \in X_{h}$. Moreover, we have $\mathbf{t}_{h} \cdot \mathbf{n}-\bar{g}_{h} \in G_{h}$. The proof of Lemma 4.6 is then reduced to estimating $\left\|\mathbf{p}_{\varepsilon}-\mathbf{t}_{h}\right\|_{X}$.

First, we obtain from (2.20) that

$$
\operatorname{div}\left(\left.\mathbf{t}_{h}\right|_{K}\right)=\gamma_{i}^{K} \operatorname{div} \overline{\mathbf{p}}_{i}^{K}=\gamma_{i}^{K} \operatorname{div} R_{i}^{K}=\operatorname{div}\left(r_{K} \mathbf{p}_{0}\right),
$$

which, along with (2.4), yields

$$
\operatorname{div}\left(\left.\mathbf{t}_{h}\right|_{K}\right)=\frac{1}{|K|} \int_{K} \operatorname{div} \mathbf{p}_{0} d x=\frac{1}{|K|} \int_{K} f d x .
$$

Thus, since $\operatorname{div} \mathbf{p}_{\varepsilon}=f$ by (2.1), we get

$$
\left\|\operatorname{div} \mathbf{p}_{\varepsilon}-\operatorname{div} \mathbf{t}_{h}\right\|_{0, \Omega} \leq C h\|f\|_{1, \Omega}
$$

It remains to estimate $\left\|\mathbf{p}_{\varepsilon}-\mathbf{t}_{h}\right\|_{0, \Omega}$, which we are going to do locally. For any $K \in \mathcal{T}_{h}$, we denote by $\widehat{r_{K} \mathbf{p}_{0}} \in R T_{0}(S)$ the extension of the linear function $r_{K} \mathbf{p}_{0} \in$ $R T_{0}(K)$ to $S$ defined as follows. Let $r_{K} \mathbf{p}_{0}=c_{i}^{K} R_{i}^{K} \in R T_{0}(K)$; then $\widehat{r_{K} \mathbf{p}_{0}}=d_{j}^{S} R_{j}^{S}$ with $d_{j}^{S}=c_{i}^{K} c_{i j}^{K}$, where $c_{i j}^{K}$ is given in (2.19). Now let $\hat{\mathbf{t}}_{h}=d_{j}^{S} \mathbf{p}_{j}^{S}$ in $S$; then $r_{S} \hat{\mathbf{t}}_{h}=d_{j}^{S} R_{j}^{S}=\widehat{r_{K} \mathbf{p}_{0}}$, where $r_{S}: H^{1}(S)^{d} \rightarrow R T_{0}(S)$ is defined in (2.10). Therefore, 
from (2.17) we know that $\hat{\mathbf{t}}_{h}=-a(x, x / \varepsilon) \nabla w_{\varepsilon}^{S}$, where $w_{\varepsilon}^{S} \in V(S)$ is the solution of the problem

$$
\begin{aligned}
\int_{S} a\left(x, \frac{x}{\varepsilon}\right) \nabla w_{\varepsilon}^{S} \nabla \varphi d x & =\int_{S} \operatorname{div}\left(\widehat{r_{K} \mathbf{p}_{0}}\right) \varphi d x \\
& -\int_{\partial S}\left(\widehat{r_{K} \mathbf{p}_{0}} \cdot \nu^{S}\right) \varphi d s \quad \forall \varphi \in H^{1}(S) .
\end{aligned}
$$

Let $w_{0}^{S} \in V(S)$ be the solution of the corresponding perturbed homogenized problem

$$
\begin{aligned}
\int_{S} \bar{a}_{S}^{*} \nabla w_{0}^{S} \nabla \varphi d x & =\int_{S} \operatorname{div}\left(\widehat{r_{K} \mathbf{p}_{0}}\right) \varphi d x \\
& -\int_{\partial S}\left(\widehat{r_{K} \mathbf{p}_{0}} \cdot \boldsymbol{\nu}^{S}\right) \varphi d s \quad \forall \varphi \in H^{1}(S),
\end{aligned}
$$

where $\bar{a}_{S}^{*}=\left(\bar{a}_{i j, S}^{*}\right) \in \mathbf{R}^{d \times d}$ is the local average of $a^{*}$ over $S$, defined as

$$
\bar{a}_{i j, S}^{*}=\frac{1}{|S|} \int_{S} a_{i j}^{*}(x) d x .
$$

Since $\widehat{r_{K} \mathbf{p}_{0}} \in R T_{0}(S)$ and $\bar{a}_{S}^{*}$ is a constant symmetric matrix, it is easy to check that

$$
\operatorname{curl}\left(\left(\bar{a}_{S}^{*}\right)^{-1} \widehat{r_{K} \mathbf{p}_{0}}\right)=0 .
$$

Hence, there exists a unique function $\Phi_{S} \in V(S)$ such that $-\bar{a}_{S}^{*} \nabla \Phi_{S}=\widehat{r_{K} \mathbf{p}_{0}}$, which implies $w_{0}^{S}=\Phi_{S}$ in $S$ by the uniqueness of the solution of the Neumann problem (4.13) in $V(S)$. We remark that this technique of "freezing coefficients" is crucial in dealing with the locally periodic coefficients.

Lemma 4.7. The solution $w_{0}^{S}$ of the problem (4.12) belongs to $H^{2}(S) \cap W^{1, \infty}(S)$ and satisfies the following stability estimates:

$$
\begin{aligned}
& \left|w_{0}^{S}\right|_{1, S} \leq C\left\|u_{0}\right\|_{1, K}, \quad\left|w_{0}^{S}\right|_{2, S} \leq C\left\|u_{0}\right\|_{2, K}, \\
& \left|w_{0}^{S}\right|_{1, \infty, S} \leq C h_{K}^{-\frac{d}{2}+1}\left\|u_{0}\right\|_{2, K}+C\left\|u_{0}\right\|_{1, \infty, K} .
\end{aligned}
$$

Moreover, the following error estimate is valid:

$$
\left\|\nabla\left(w_{0}^{S}-u_{0}\right)\right\|_{0, K} \leq C h_{K}\left\|u_{0}\right\|_{2, K} .
$$

Proof. Since $-\bar{a}_{S}^{*} \nabla w_{0}^{S}=\widehat{r_{K} \mathbf{p}_{0}}$ and $\widehat{r_{K} \mathbf{p}_{0}} \in L^{\infty}(S)$, it is obvious that $w_{0}^{S} \in H^{2}(S)$ $\cap W^{1, \infty}(S)$. By (2.11) and the assumption $h_{S} \leq C_{0} h_{K}$, we have

$$
\begin{aligned}
\left\|\nabla\left(w_{0}^{S}-u_{0}\right)\right\|_{0, K} & \leq C\left\|r_{K} \mathbf{p}_{0}-\mathbf{p}_{0}\right\|_{0, K}+\left\|\left(a^{*}-\bar{a}_{S}^{*}\right) \nabla u_{0}\right\|_{0, K} \\
& \leq C h_{K}\left|\mathbf{p}_{0}\right|_{1, K}+C h_{K}\left\|\nabla u_{0}\right\|_{0, K} \leq C h_{K}\left\|u_{0}\right\|_{2, K},
\end{aligned}
$$

and

$$
\begin{aligned}
& \left|w_{0}^{S}\right|_{1, K} \leq C\left\|\widehat{r_{K} \mathbf{p}_{0}}\right\|_{0, S} \leq C\left\|r_{K} \mathbf{p}_{0}\right\|_{0, K} \leq C\left\|\mathbf{p}_{0}\right\|_{0, K} \leq C\left\|u_{0}\right\|_{1, K}, \\
& \left|w_{0}^{S}\right|_{2, K} \leq C\left|\widehat{r_{K} \mathbf{p}_{0}}\right|_{1, S} \leq C\left|r_{K} \mathbf{p}_{0}\right|_{1, K} \leq C\left|\mathbf{p}_{0}\right|_{1, K} \leq C\left\|u_{0}\right\|_{2, K} .
\end{aligned}
$$


Next, let $P_{K} \mathbf{p}_{0}=\frac{1}{|K|} \int_{K} \mathbf{p}_{0} d x$; then by an inverse estimate and (2.11) we obtain that

$$
\begin{aligned}
\left|w_{0}^{S}\right|_{1, \infty, K} \leq C\left\|\widehat{r_{K} \mathbf{p}_{0}}\right\|_{0, \infty, S} & \leq C\left\|r_{K} \mathbf{p}_{0}\right\|_{0, \infty, K} \\
& \leq C\left\|r_{K} \mathbf{p}_{0}-P_{K} \mathbf{p}_{0}\right\|_{0, \infty, K}+C\left\|P_{K} \mathbf{p}_{0}\right\|_{0, \infty, K} \\
& \leq C h_{K}^{-\frac{d}{2}}\left\|r_{K} \mathbf{p}_{0}-P_{K} \mathbf{p}_{0}\right\|_{0, K}+C\left\|P_{K} \mathbf{p}_{0}\right\|_{0, \infty, K} \\
& \leq C h_{K}^{-\frac{d}{2}+1}\left\|u_{0}\right\|_{2, K}+C\left\|u_{0}\right\|_{1, \infty, K} .
\end{aligned}
$$

This completes the proof.

Lemma 4.8. Let $w_{\varepsilon}^{S}$ be the solution of (4.12) and $w_{1}^{S}=w_{0}^{S}-\varepsilon \chi^{j}\left(x, \frac{x}{\varepsilon}\right) \frac{\partial w_{0}^{S}}{\partial x_{j}}$. Then there exists a constant $C$, independent of $h$ and $\varepsilon$, such that

$$
\left\|\nabla w_{\varepsilon}^{S}-\nabla w_{1}^{S}\right\|_{0, K} \leq C \varepsilon\left\|u_{0}\right\|_{2, K}+C\left(h_{K}+\varepsilon\right)\left\|u_{0}\right\|_{1, K}+C \varepsilon h_{K}^{\frac{d}{2}-1}\left\|u_{0}\right\|_{1, \infty, K} .
$$

Proof. Note that in the definition of the homogenized problem (4.13) we have used the constant coefficient $\bar{a}_{S}^{*}$ instead of $a^{*}(x)$. Thus, the homogenization result in Theorem 3.1 cannot be directly used. However, if we let $\theta_{\varepsilon}^{S} \in H^{1}(S) / \mathbf{R}$ be the solution of the problem

$$
\int_{S} a\left(x, \frac{x}{\varepsilon}\right) \nabla \theta_{\varepsilon}^{S} \nabla \varphi d x=\int_{S} \frac{\partial}{\partial x_{j}}\left(\alpha_{i j}^{k}\left(x, \frac{x}{\varepsilon}\right) \frac{\partial w_{0}^{S}}{\partial x_{k}}\right) \frac{\partial \varphi}{\partial x_{i}} d x \quad \forall \varphi \in H^{1}(S),
$$

then, using an argument similar to the proof of Theorem 3.1] we obtain from (4.12)(4.13) that

$$
\begin{aligned}
\int_{S} a( & \left.x, \frac{x}{\varepsilon}\right) \nabla\left(w_{\varepsilon}^{S}-w_{1}^{S}-\varepsilon \theta_{\varepsilon}^{S}\right) \nabla \varphi d x \\
= & \int_{S}\left(\bar{a}_{S}^{*}-a^{*}(x)\right) \nabla w_{0}^{S} \nabla \varphi d x \\
& +\varepsilon \int_{S}\left[a_{i j}\left(x, \frac{x}{\varepsilon}\right) \chi^{k}\left(x, \frac{x}{\varepsilon}\right) \frac{\partial^{2} w_{0}^{S}}{\partial x_{j} \partial x_{k}}+a_{i j}\left(x, \frac{x}{\varepsilon}\right) \frac{\partial \chi^{k}}{\partial x_{j}}\left(x, \frac{x}{\varepsilon}\right) \frac{\partial w_{0}^{S}}{\partial x_{k}}\right] \frac{\partial \varphi}{\partial x_{i}} d x \\
& -\varepsilon \int_{S}\left[\alpha_{i j}^{k}\left(x, \frac{x}{\varepsilon}\right) \frac{\partial^{2} w_{0}^{S}}{\partial x_{j} \partial x_{k}}+\frac{\partial \alpha_{i j}^{k}}{\partial x_{j}}\left(x, \frac{x}{\varepsilon}\right) \frac{\partial w_{0}^{S}}{\partial x_{k}}\right] \frac{\partial \varphi}{\partial x_{i}} d x \quad \forall \varphi \in H^{1}(S) \\
= & : \mathrm{I}+\mathrm{II}+\mathrm{III} .
\end{aligned}
$$

From the definition of $\bar{a}_{S}^{*}$ in (4.13) we know that $\left\|\bar{a}_{S}^{*}-a^{*}\right\|_{0, \infty, S} \leq C h_{S}\left|a^{*}\right|_{1, \infty, S} \leq$ $C h_{S} \leq C h_{K}$. Hence

$$
\mathrm{I} \leq C h_{K}\left|w_{0}^{S}\right|_{1, S}\|\nabla \varphi\|_{0, S} .
$$

Next, since $a_{i j}(x, y), \chi^{k}(x, y),\left(\partial \chi^{k} / \partial x_{j}\right)(x, y), \alpha_{i j}^{k}(x, y)$ and $\left(\partial \alpha_{i j}^{k} / \partial x_{j}\right)(x, y)$ are bounded functions in $\bar{\Omega} \times \bar{Y}$, we deduce that

$$
\mathrm{II}+\mathrm{III} \leq C \varepsilon\left(\left|w_{0}^{S}\right|_{2, S}+\left|w_{0}^{S}\right|_{1, S}\right)\|\nabla \varphi\|_{0, S} .
$$

By taking $\varphi=w_{\varepsilon}^{S}-w_{1}^{S}-\varepsilon \theta_{\varepsilon}^{S}$ in (4.14) and using (4.15), (4.16), we have that

$$
\begin{aligned}
\left\|\nabla\left(w_{\varepsilon}^{S}-w_{1}^{S}-\varepsilon \theta_{\varepsilon}^{S}\right)\right\|_{0, S} & \leq C \varepsilon\left|w_{0}^{S}\right|_{2, S}+C\left(h_{K}+\varepsilon\right)\left|w_{0}^{S}\right|_{1, S} \\
& \leq C \varepsilon\left\|u_{0}\right\|_{2, K}+C\left(h_{K}+\varepsilon\right)\left\|u_{0}\right\|_{1, K},
\end{aligned}
$$


where we have used Lemma 4.7] in the second inequality. Finally, by (4.9) and Lemma 4.7] we have, since $\operatorname{dist}(\partial K, \partial S) \geq \delta_{0} h_{K}$ from (H3),

$$
\left\|\varepsilon \nabla \theta_{\varepsilon}^{S}\right\|_{0, K} \leq C \varepsilon h_{K}^{\frac{d}{2}-1}\left|w_{0}^{S}\right|_{1, \infty, S} \leq C \varepsilon\left\|u_{0}\right\|_{2, K}+C \varepsilon h_{K}^{\frac{d}{2}-1}\left\|u_{0}\right\|_{1, \infty, K} .
$$

This completes the proof.

Now we are ready to complete the proof of Lemma 4.6 .

Proof of Lemma 4.6. Let $u_{1}^{\varepsilon}=u_{0}-\varepsilon \chi^{j}\left(x, \frac{x}{\varepsilon}\right) \frac{\partial u_{0}}{\partial x_{j}}$. Then it is easy to show by Lemma 4.7 that

$$
\left\|\nabla w_{1}^{S}-\nabla u_{1}^{\varepsilon}\right\|_{0, K} \leq C\left(h_{K}+\varepsilon\right)\left\|u_{0}\right\|_{2, K}
$$

Thus, we have, by Lemma 4.8 ,

$$
\begin{aligned}
\| \mathbf{p}_{\varepsilon} & -\mathbf{t}_{h} \|_{0, K} \\
& \leq C\left\|\nabla u_{\varepsilon}-\nabla w_{\varepsilon}^{S}\right\|_{0, K} \\
& \leq C\left\|\nabla u_{\varepsilon}-\nabla u_{1}^{\varepsilon}\right\|_{0, K}+C\left\|\nabla u_{1}^{\varepsilon}-\nabla w_{1}^{S}\right\|_{0, K}+C\left\|\nabla w_{1}^{S}-w_{\varepsilon}^{S}\right\|_{0, K} \\
& \leq C\left\|\nabla u_{\varepsilon}-\nabla u_{1}^{\varepsilon}\right\|_{0, K}+C\left(h_{K}+\varepsilon\right)\left\|u_{0}\right\|_{2, K}+C \varepsilon h_{K}^{\frac{d}{2}-1}\left\|u_{0}\right\|_{1, \infty, K} .
\end{aligned}
$$

Finally, by Theorem 3.1 we arrive at

$$
\left\|\mathbf{p}_{\varepsilon}-\mathbf{t}_{h}\right\|_{0, \Omega} \leq C(h+\varepsilon)\left\|u_{0}\right\|_{2, \Omega}+C\left(\frac{\varepsilon}{h}+\sqrt{\varepsilon}\right)\left\|u_{0}\right\|_{1, \infty, \Omega} .
$$

This estimate, together with (4.10), completes the proof.

To estimate the nonconforming error in (4.7), we first recall the following result, whose proof can be found in [13, Lemma 3.2].

Lemma 4.9. Let $N \in L^{\infty}\left(\mathbf{R}^{d}\right)$ be a periodic function with respect to the unit cube $Y$ and assume that its average over $Y$ vanishes. Then, for any $\zeta \in H^{1}(K) \cap L^{\infty}(K)$, $K \in \mathcal{T}_{h}$, we have

$$
\left|\int_{K} \zeta(x) N\left(x, \frac{x}{\varepsilon}\right) d x\right| \leq C \varepsilon h_{K}^{\frac{d}{2}}\|\nabla \zeta\|_{0, K}+C \varepsilon h_{K}^{d-1}\|\zeta\|_{0, \infty, K} .
$$

For any $\mathbf{q}_{h} \in X_{h}$ and $K \in \mathcal{T}_{h}$, since $\left.\mathbf{q}_{h}\right|_{K} \in \Lambda(K)$, there exist constants $\beta_{i}^{K}$ such that $\left.\mathbf{q}_{h}\right|_{K}=\beta_{i}^{K} \overline{\mathbf{p}}_{i}^{K}$, and thus $\left.\mathbf{q}_{h}\right|_{K}=\left.\beta_{i}^{K} c_{i j}^{K} \mathbf{p}_{j}^{S}\right|_{K}$ by (2.18). Let $\hat{\mathbf{q}}_{h}=\beta_{i}^{K} c_{i j}^{K} \mathbf{p}_{j}^{S}$ in $S$; then we have $r_{S} \hat{\mathbf{q}}_{h}=\beta_{i}^{K} c_{i j}^{K} R_{j}^{S}$, where $r_{S}: H^{1}(S)^{d} \rightarrow R T_{0}(S)$ is defined in (2.10). From 2.19) we have $\Pi_{h} \mathbf{q}_{h}=\left.r_{S} \hat{\mathbf{q}}_{h}\right|_{K}$ in $K$. Now from the definition of the basis function $\mathbf{p}_{j}^{S} \in \Sigma(S)$ in (2.17) we know that $\hat{\mathbf{q}}_{h}=-a(x, x / \varepsilon) \nabla W_{\varepsilon}^{S}$ in $S$, where $W_{\varepsilon}^{S} \in V(S)$ is the solution of the problem

$$
\begin{aligned}
\int_{S} a\left(x, \frac{x}{\varepsilon}\right) \nabla W_{\varepsilon}^{S} \nabla \varphi d x & =\int_{S} \operatorname{div}\left(r_{S} \hat{\mathbf{q}}_{h}\right) \varphi d x \\
& -\int_{\partial S}\left(r_{S} \hat{\mathbf{q}}_{h} \cdot \boldsymbol{\nu}^{S}\right) \varphi d s \quad \forall \varphi \in H^{1}(S) .
\end{aligned}
$$

Let $W_{0}^{S} \in V(S)$ be the solution of the corresponding perturbed homogenized problem

$$
\begin{aligned}
\int_{S} \bar{a}_{S}^{*} \nabla W_{0}^{S} \nabla \varphi d x & =\int_{S} \operatorname{div}\left(r_{S} \hat{\mathbf{q}}_{h}\right) \varphi d x \\
& -\int_{\partial S}\left(r_{S} \hat{\mathbf{q}}_{h} \cdot \boldsymbol{\nu}^{S}\right) \varphi d s \quad \forall \varphi \in H^{1}(S),
\end{aligned}
$$


where $\bar{a}_{S}^{*}=\left(\bar{a}_{i j, S}^{*}\right) \in \mathbf{R}^{d \times d}$ with

$$
\bar{a}_{i j, S}^{*}=\frac{1}{|S|} \int_{S} a_{i j}^{*}(x) d x .
$$

Again, the argument before Lemma4.7implies that $r_{S} \hat{\mathbf{q}}_{h}=-\bar{a}_{S}^{*} \nabla W_{0}^{S}$ in $S$. By the finite element inverse estimates and the assumption $h_{S} \leq C h_{K}$, we easily obtain

$$
\left|W_{0}^{S}\right|_{1, \infty, S} \leq C h_{K}^{-\frac{d}{2}}\left|W_{0}^{S}\right|_{1, K}, \quad\left|W_{0}^{S}\right|_{m, S} \leq C h_{K}^{1-m}\left|W_{0}^{S}\right|_{1, K}, \quad m=1,2 .
$$

Let $W_{1}^{S}=W_{0}^{S}-\varepsilon \chi^{j}\left(x, \frac{x}{\varepsilon}\right) \frac{\partial W_{0}^{S}}{\partial x_{j}}$. Then, using an argument similar to the proof of Lemma 4.8 we deduce that

$$
\begin{aligned}
& \left\|\nabla W_{\varepsilon}^{S}-\nabla W_{1}^{S}\right\|_{0, K} \\
& \quad \leq C \varepsilon\left|W_{0}^{S}\right|_{2, S}+C\left(h_{K}+\varepsilon\right)\left|W_{0}^{S}\right|_{1, S}+C \varepsilon h_{K}^{\frac{d}{2}-1}\left|W_{0}^{S}\right|_{1, \infty, S} \\
& \quad \leq C \varepsilon\left|W_{0}^{S}\right|_{2, S}+C\left(h_{K}+\varepsilon+\varepsilon h_{K}^{-1}\right)\left|W_{0}^{S}\right|_{1, S},
\end{aligned}
$$

where we have used inverse estimate in the last inequality. Now we are going to prove the following stability estimate, which plays an important role in the subsequent analysis.

Lemma 4.10. Under the assumptions (H1)-(H4) there exist constants $\delta>0$ and $C>0$, independent of $h$ and $\varepsilon$, such that if $h_{K} \leq \delta$ and $\varepsilon / h_{K} \leq \delta$ for all $K \in \mathcal{T}_{h}$, the following stability estimate is valid:

$$
\left|W_{0}^{S}\right|_{1, K} \leq C\left\|\mathbf{q}_{h}\right\|_{0, K} \quad \forall K \in \mathcal{T}_{h} .
$$

Proof. From (2.3), (4.20), and (4.19) we have

$$
\begin{aligned}
\left\|\mathbf{q}_{h}\right\|_{0, K} & =\left\|a \nabla W_{\varepsilon}^{S}\right\|_{0, K} \\
& \geq C\left\|\nabla W_{\varepsilon}^{S}\right\|_{0, K} \\
& \geq C\left\|\nabla W_{1}^{S}\right\|_{0, K}-C \varepsilon\left|W_{0}^{S}\right|_{2, S}-C\left(h_{K}+\varepsilon+\varepsilon h_{K}^{-1}\right)\left|W_{0}^{S}\right|_{1, S} \\
& \geq C\left\|\nabla W_{1}^{S}\right\|_{0, K}-C\left(h_{K}+\varepsilon+\varepsilon h_{K}^{-1}\right)\left|W_{0}^{S}\right|_{1, K} .
\end{aligned}
$$

Next, by simple calculations we get

$$
\begin{aligned}
a_{i j}\left(x, \frac{x}{\varepsilon}\right) \frac{\partial W_{1}^{S}}{\partial x_{j}}= & a_{i j}^{*}(x) \frac{\partial W_{0}^{S}}{\partial x_{j}}-G_{i}^{j}\left(x, \frac{x}{\varepsilon}\right) \frac{\partial W_{0}^{S}}{\partial x_{j}} \\
& -\varepsilon a_{i j}\left(x, \frac{x}{\varepsilon}\right) \chi^{k}\left(x, \frac{x}{\varepsilon}\right) \frac{\partial^{2} W_{0}^{S}}{\partial x_{j} \partial x_{k}} \\
& -\varepsilon a_{i j}\left(x, \frac{x}{\varepsilon}\right) \frac{\partial \chi^{k}}{\partial x_{j}}\left(x, \frac{x}{\varepsilon}\right) \frac{\partial W_{0}^{S}}{\partial x_{k}},
\end{aligned}
$$

where $G_{i}^{j}(x, y)$ is given by

$$
G_{i}^{j}(x, y)=a_{i j}^{*}(x)-a_{i k}(x, y)\left(\delta_{k j}-\frac{\partial \chi^{j}}{\partial y_{k}}(x, y)\right) .
$$


Multiplying the above equality by $\nabla W_{0}^{S}$ and then integrating over $K$, we obtain

$$
\begin{aligned}
\int_{K} a^{*} & \nabla W_{0}^{S} \cdot \nabla W_{0}^{S} d x \\
= & \int_{K} a\left(x, \frac{x}{\varepsilon}\right) \nabla W_{1}^{S} \cdot \nabla W_{0}^{S} d x \\
& +\int_{K} G_{i}^{j}\left(x, \frac{x}{\varepsilon}\right) \frac{\partial W_{0}^{S}}{\partial x_{j}} \frac{\partial W_{0}^{S}}{\partial x_{i}} d x \\
& +\varepsilon \int_{K} a_{i j}\left(x, \frac{x}{\varepsilon}\right) \chi^{k}\left(x, \frac{x}{\varepsilon}\right) \frac{\partial^{2} W_{0}^{S}}{\partial x_{j} \partial x_{k}} \frac{\partial W_{0}^{S}}{\partial x_{i}} d x \\
& +\varepsilon \int_{K} a_{i j}\left(x, \frac{x}{\varepsilon}\right) \frac{\partial \chi^{k}}{\partial x_{j}}\left(x, \frac{x}{\varepsilon}\right) \frac{\partial W_{0}^{S}}{\partial x_{k}} \frac{\partial W_{0}^{S}}{\partial x_{i}} d x .
\end{aligned}
$$

Now let $g_{i j}^{K}(y)=\frac{1}{|K|} \int_{K} G_{i}^{j}(x, y) d x$; then $g_{i j}^{K}$ is periodic with respect to the unit cube $Y$ and $\int_{Y} g_{i j}^{K}(y) d y=0$. Moreover, by finite element interpolation theory, we have

$$
\left\|g_{i j}^{K}(y)-G_{i}^{j}(\cdot, y)\right\|_{0, \infty, K} \leq C h_{K}\left\|\nabla_{x} G(\cdot, y)\right\|_{0, \infty, K} \leq C h_{K}, \quad \forall y \in \mathbf{R}^{d} .
$$

Now by Lemma 4.9 and (4.19) we know that

$$
\begin{aligned}
\left|\int_{K} G_{i}^{j}\left(x, \frac{x}{\varepsilon}\right) \frac{\partial W_{0}^{S}}{\partial x_{j}} \frac{\partial W_{0}^{S}}{\partial x_{i}} d x\right| \\
=\left|\int_{K}\left(G_{i}^{j}\left(x, \frac{x}{\varepsilon}\right)-g_{i j}^{K}\left(\frac{x}{\varepsilon}\right)\right) \frac{\partial W_{0}^{S}}{\partial x_{j}} \frac{\partial W_{0}^{S}}{\partial x_{i}} d x\right| \\
\quad+\left|\int_{K} g_{i j}^{K}\left(\frac{x}{\varepsilon}\right) \frac{\partial W_{0}^{S}}{\partial x_{j}} \frac{\partial W_{0}^{S}}{\partial x_{i}} d x\right| \\
\leq C h_{K}\left|W_{0}^{S}\right|_{1, K}^{2}+C \varepsilon h_{K}^{\frac{d}{2}}\left|W_{0}^{S}\right|_{2, K}\left|W_{0}^{S}\right|_{1, \infty, K}+C \varepsilon h_{K}^{-1}\left|W_{0}^{S}\right|_{1, K}^{2} \\
\leq C\left(h_{K}+\varepsilon h_{K}^{-1}\right)\left|W_{0}^{S}\right|_{1, K}^{2} .
\end{aligned}
$$

Moreover, the estimates in (4.19) imply that

$$
\begin{aligned}
& \left|\varepsilon \int_{K} a_{i j}\left(x, \frac{x}{\varepsilon}\right) \chi^{K}\left(x, \frac{x}{\varepsilon}\right) \frac{\partial^{2} W_{0}^{S}}{\partial x_{j} \partial x_{k}} \frac{\partial W_{0}^{S}}{\partial x_{i}} d x\right| \leq C \varepsilon h_{K}^{-1}\left|W_{0}^{S}\right|_{1, K}^{2}, \\
& \left|\varepsilon \int_{K} a_{i j}\left(x, \frac{x}{\varepsilon}\right) \frac{\partial \chi^{k}}{\partial x_{j}}\left(x, \frac{x}{\varepsilon}\right) \frac{\partial W_{0}^{S}}{\partial x_{k}} \frac{\partial W_{0}^{S}}{\partial x_{i}} d x\right| \leq C \varepsilon\left|W_{0}^{S}\right|_{1, K}^{2} .
\end{aligned}
$$

Substituting these estimates into (4.23), we conclude that

$$
\left\|\nabla W_{1}^{S}\right\|_{0, K} \geq C\left|W_{0}^{S}\right|_{1, K}-C\left(\varepsilon+h_{K}+\varepsilon h_{K}^{-1}\right)\left|W_{0}^{S}\right|_{1, K} .
$$

Now the desired estimate follows from (4.21) under the condition that $\varepsilon / h_{K}$ is sufficiently small. This complete the proof.

From this lemma and (4.19), we deduce that

$$
\left|W_{0}^{S}\right|_{1, S} \leq C\left\|\mathbf{q}_{h}\right\|_{0, K} \quad \text { and } \quad\left|W_{0}^{S}\right|_{1, \infty, S} \leq C h_{K}^{-\frac{d}{2}}\left\|\mathbf{q}_{h}\right\|_{0, K}
$$

Moreover, since $-\bar{a}_{S}^{*} \nabla W_{0}^{S}=r_{S} \hat{\mathbf{q}}_{h} \in R T_{0}(S)$ and $\operatorname{div} r_{S} \hat{\mathbf{q}}_{h}=\operatorname{div} \mathbf{q}_{h}$ in $K$, we have

$$
\begin{aligned}
\left|W_{0}^{S}\right|_{2, S} \leq C\left|r_{S} \hat{\mathbf{q}}_{h}\right|_{1, S} & =C\left\|\operatorname{div} s r_{S} \hat{\mathbf{q}}_{h}\right\|_{0, S} \\
& =C\left\|\operatorname{div} \hat{\mathbf{q}}_{h}\right\|_{0, S} \leq C\left\|\operatorname{div} \mathbf{q}_{h}\right\|_{0, K} .
\end{aligned}
$$


Proof of Theorem 2.2. By Lemmas 4.5 and 4.6 we know that we only need to estimate the nonconforming error in Lemma 4.4 Since $\operatorname{div} \Pi_{h} \mathbf{q}_{h}=\operatorname{div} \mathbf{q}_{h}$ by definition, we have $b_{h}\left(\mathbf{q}_{h}, u_{\varepsilon}\right)=b_{h}\left(\Pi_{h} \mathbf{q}_{h}, u_{\varepsilon}\right)$ for any $\mathbf{q}_{h} \in G_{h}$. Thus, since $\Pi_{h} \mathbf{q}_{h} \in W_{h} \cap H_{0}(\operatorname{div} ; \Omega)$, we get from (2.9) that

$$
\begin{aligned}
a_{h}\left(\mathbf{p}_{\varepsilon}, \mathbf{q}_{h}\right)+b_{h}\left(\mathbf{q}_{h}, u_{\varepsilon}\right) & =a_{h}\left(\mathbf{p}_{\varepsilon}, \mathbf{q}_{h}-\Pi_{h} \mathbf{q}_{h}\right)+b_{h}\left(\mathbf{q}_{h}-\Pi_{h} \mathbf{q}_{h}, u_{\varepsilon}\right) \\
& =a_{h}\left(\mathbf{p}_{\varepsilon}, \mathbf{q}_{h}-\Pi_{h} \mathbf{q}_{h}\right) \\
& =-\sum_{K \in \mathcal{T}_{h}} \int_{K} \nabla u_{\varepsilon} \cdot\left(\mathbf{q}_{h}-\Pi_{h} \mathbf{q}_{h}\right) d x, \quad \forall \mathbf{q}_{h} \in G_{h} .
\end{aligned}
$$

For any $\mathbf{q}_{h} \in X_{h}$ and $K \in \mathcal{T}_{h}$, since $\mathbf{q}_{h}=-a(x, x / \varepsilon) \nabla W_{\varepsilon}^{S}$ in $K$ and $\Pi_{h} \mathbf{q}_{h}=$ $-\bar{a}_{S}^{*} \nabla W_{0}^{S}$ in $K$ with $W_{\varepsilon}^{S}, W_{0}^{S} \in V(S)$ the respective solutions of (4.18) and (4.19), we deduce from (4.22) that

$$
\begin{aligned}
\int_{K} \nabla u_{\varepsilon} \cdot\left(\mathbf{q}_{h}-\Pi_{h} \mathbf{q}_{h}\right) d x & =-\int_{K} \nabla u_{\varepsilon} \cdot a\left(x, \frac{x}{\varepsilon}\right) \nabla\left(W_{\varepsilon}^{S}-W_{1}^{S}\right) d x \\
& +\int_{K} \nabla u_{\varepsilon} \cdot\left(\bar{a}_{S}^{*}-a^{*}\right) \nabla W_{0}^{S} d x \\
& +\int_{K} G_{i}^{j}\left(x, \frac{x}{\varepsilon}\right) \frac{\partial u_{\varepsilon}}{\partial x_{i}} \frac{\partial W_{0}^{S}}{\partial x_{j}} d x \\
& +\varepsilon \int_{K} a_{i j}\left(x, \frac{x}{\varepsilon}\right) \frac{\partial \chi^{k}}{\partial x_{j}}\left(x, \frac{x}{\varepsilon}\right) \frac{\partial u_{\varepsilon}}{\partial x_{i}} \frac{\partial W_{0}^{S}}{\partial x_{k}} d x \\
& +\varepsilon \int_{K} a_{i k}\left(x, \frac{x}{\varepsilon}\right) \chi^{j}\left(x, \frac{x}{\varepsilon}\right) \frac{\partial u_{\varepsilon}}{\partial x_{i}} \frac{\partial^{2} W_{0}^{S}}{\partial x_{j} \partial x_{k}} d x \\
& :=\mathrm{I}+\cdots+\mathrm{V} .
\end{aligned}
$$

Now we are going to estimate the terms I, $\cdots$, V, term by term. First, by (4.20) and (4.25)-(4.27) we get

$$
\begin{aligned}
|\mathrm{I}| & \leq C\left\|\nabla u_{\varepsilon}\right\|_{0, K}\left\|\nabla\left(W_{\varepsilon}^{S}-W_{1}^{S}\right)\right\|_{0, K} \\
& \leq C\left(\varepsilon+h_{K}+\varepsilon h_{K}^{-1}\right)\left\|\nabla u_{\varepsilon}\right\|_{0, K}\left\|\mathbf{q}_{h}\right\|_{\text {div }, K} .
\end{aligned}
$$

Next, by (4.25)-(4.27) and Lemma 4.10, we obtain

$$
|\mathrm{II}|+|\mathrm{IV}|+|\mathrm{V}| \leq C\left(\varepsilon+h_{K}\right)\left\|\nabla u_{\varepsilon}\right\|_{0, K}\left\|\mathbf{q}_{h}\right\|_{\mathrm{div}, K} .
$$

To estimate the term III, we write $u_{1}^{\varepsilon}=u_{0}+\varepsilon \chi^{j}\left(x, \frac{x}{\varepsilon}\right) \frac{\partial u_{0}}{\partial x_{j}}$ and derive

$$
\begin{aligned}
\mathrm{III} & =\int_{K} G_{i}^{j}\left(x, \frac{x}{\varepsilon}\right) \frac{\partial\left(u_{\varepsilon}-u_{1}^{\varepsilon}\right)}{\partial x_{i}} \frac{\partial W_{0}^{S}}{\partial x_{j}} d x \\
& +\int_{K} G_{i}^{j}\left(x, \frac{x}{\varepsilon}\right) \frac{\partial u_{0}}{\partial x_{i}} \frac{\partial W_{0}^{S}}{\partial x_{j}} d x \\
& +\int_{K} G_{i}^{j}\left(x, \frac{x}{\varepsilon}\right) \frac{\partial \chi^{k}}{\partial y_{i}}\left(x, \frac{x}{\varepsilon}\right) \frac{\partial u_{0}}{\partial x_{k}} \frac{\partial W_{0}^{S}}{\partial x_{j}} d x \\
& +\varepsilon \int_{K} G_{i}^{j}\left(x, \frac{x}{\varepsilon}\right) \chi^{k}\left(x, \frac{x}{\varepsilon}\right) \frac{\partial^{2} u_{0}}{\partial x_{i} \partial x_{k}} \frac{\partial W_{0}^{S}}{\partial x_{j}} d x \\
& +\varepsilon \int_{K} G_{i}^{j}\left(x, \frac{x}{\varepsilon}\right) \frac{\partial \chi^{k}}{\partial x_{i}}\left(x, \frac{x}{\varepsilon}\right) \frac{\partial u_{0}}{\partial x_{k}} \frac{\partial W_{0}^{S}}{\partial x_{j}} d x \\
& :=\operatorname{III}_{1}+\cdots+\mathrm{III}_{5} .
\end{aligned}
$$


By Cauchy's inequality and (4.25) we obtain

$$
\left|\mathrm{III}_{1}\right| \leq C\left\|\nabla u_{\varepsilon}-\nabla u_{1}^{\varepsilon}\right\|_{0, K}\left\|\nabla W_{0}^{S}\right\|_{0, K} \leq C\left\|\nabla u_{\varepsilon}-\nabla u_{1}^{\varepsilon}\right\|_{0, K}\left\|\mathbf{q}_{h}\right\|_{0, K} .
$$

Next, by the argument used in deriving (4.24) and by Lemma 4.9. we have

$$
\left|\mathrm{III}_{2}\right| \leq C\left(\varepsilon+h_{K}\right)\left\|u_{0}\right\|_{2, K}\left\|\mathbf{q}_{h}\right\|_{\operatorname{div}, K}+C \varepsilon h_{K}^{\frac{d}{2}-1}\left\|u_{0}\right\|_{1, \infty, K}\left\|\mathbf{q}_{h}\right\|_{\operatorname{div}, K} .
$$

Since $\partial G_{i}^{j}(x, y) / \partial y_{i}=0$ in $Y$, integration by parts gives

$$
\int_{Y} G_{i}^{j}(x, y) \frac{\partial \chi^{k}}{\partial y_{i}}(x, y) d y=0 .
$$

Thus, again by the argument used in deriving 4.24) and by Lemma 4.9, we get

$$
\left|\mathrm{III}_{3}\right| \leq C\left(\varepsilon+h_{K}\right)\left\|u_{0}\right\|_{2, K}\left\|\mathbf{q}_{h}\right\|_{\operatorname{div}, K}+C \varepsilon h_{K}^{\frac{d}{2}-1}\left\|u_{0}\right\|_{1, \infty, K}\left\|\mathbf{q}_{h}\right\|_{\operatorname{div}, K} .
$$

Finally, by Cauchy's inequality and (4.25), we have

$$
\left|\mathrm{III}_{4}\right|+\left|\mathrm{III}_{5}\right| \leq C \varepsilon\left\|u_{0}\right\|_{2, K}\left\|\mathbf{q}_{h}\right\|_{0, K} .
$$

Combining the above estimates, we conclude that

$$
\begin{aligned}
\sum_{K \in \mathcal{T}_{h}} & \int_{K} \nabla u_{\varepsilon} \cdot\left(\mathbf{q}_{h}-\Pi_{h} \mathbf{q}_{h}\right) d x \\
\leq & C\left(\varepsilon+h+\frac{\varepsilon}{h}\right)\left\|\nabla u_{\varepsilon}\right\|_{0, \Omega}\left\|\mathbf{q}_{h}\right\|_{X}+C\left\|\nabla u_{\varepsilon}-\nabla u_{1}^{\varepsilon}\right\|_{0, \Omega}\left\|\mathbf{q}_{h}\right\|_{X} \\
& +C(\varepsilon+h)\left\|u_{0}\right\|_{2, \Omega}\left\|\mathbf{q}_{h}\right\|_{X}+C \frac{\varepsilon}{h}\left\|u_{0}\right\|_{1, \infty, \Omega}\left\|\mathbf{q}_{h}\right\|_{X},
\end{aligned}
$$

which, after using Theorem 3.1, yields

$$
\begin{aligned}
\sup _{\mathbf{q}_{h} \in G_{h}} & \frac{\left|a_{h}\left(\mathbf{p}_{\varepsilon}, \mathbf{q}_{h}\right)+b_{h}\left(\mathbf{q}_{h}, u_{\varepsilon}\right)\right|}{\left\|\mathbf{q}_{h}\right\|_{X}} \\
\leq & C(\varepsilon+h)\left(\left\|u_{0}\right\|_{2, \Omega}+\|f\|_{0, \Omega}+\left\|\mathbf{q}_{0}\right\|_{\operatorname{div}, \Omega}\right) \\
& +C\left(\frac{\varepsilon}{h}+\sqrt{\varepsilon}\right)\left(\|f\|_{0, \Omega}+\left\|\mathbf{q}_{0}\right\|_{\operatorname{div}, \Omega}+\left\|u_{0}\right\|_{1, \infty, \Omega}\right) .
\end{aligned}
$$

This completes the proof.

To conclude this section, we remark that in the proofs of Lemma4.3 and Theorem 2.2, the choice of the constants $c_{i j}^{K}$ through the relation $R_{i}^{K}=\left.c_{i j}^{K} R_{j}^{S}\right|_{K}$ in (2.18) to define the over-sampling multiscale bases plays a crucial role. Another natural method to define the over-sampling multiscale bases is $\tilde{\mathbf{p}}_{i}^{K}=\left.\tilde{c}_{i j}^{K} \mathbf{p}_{j}^{S}\right|_{K}$ in $K$ with the coefficients $\tilde{c}_{i j}^{K}$ so chosen that

$$
\int_{e_{j}^{K}} \tilde{\mathbf{p}}_{i}^{K} \cdot \boldsymbol{\nu}^{K} d s=\left\{\begin{array}{lll}
1 & \text { if } & j=i \\
0 & \text { if } & j \neq i .
\end{array}\right.
$$

We have carried out numerical experiments for both methods to define the oversampling multiscale bases and found that both methods perform quite well. But a rigorous theoretical justification of the choice (4.27), i.e., the derivation of an error estimate like that in Theorem 2.2 is left for future study. 

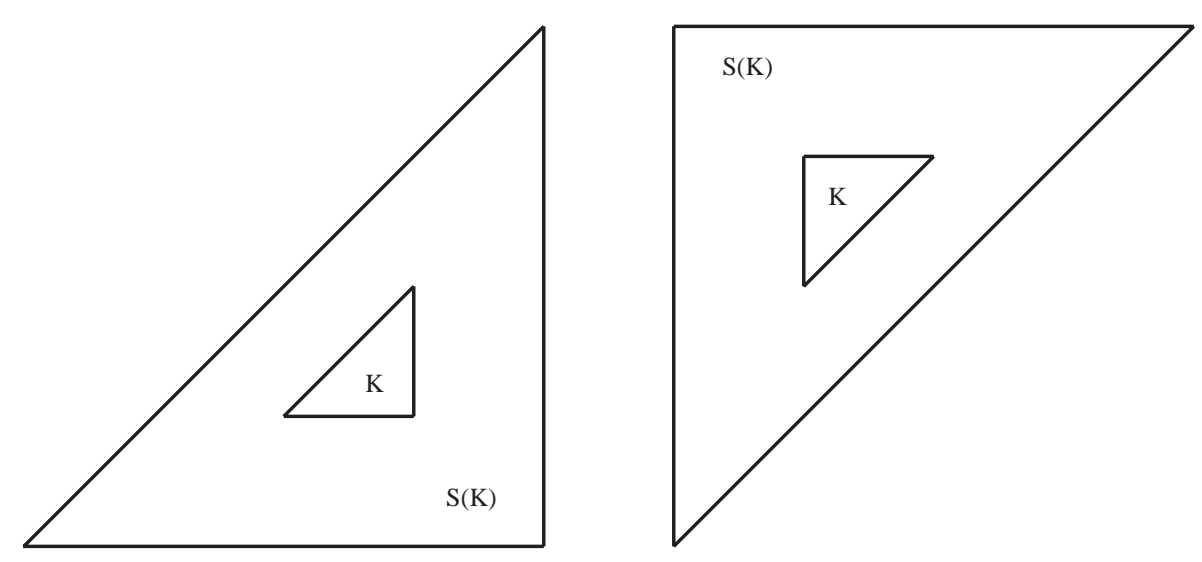

Figure 1. The element $K$ and its over-sampling element $S(K)$ : lower right elements (left) and upper right elements (right). The length of the horizontal and vertical edges of $S(K)$ is four times the corresponding length of the edges of $K$.

\section{Numerical Results}

In this section we first test the accuracy of the over-sampling mixed multiscale finite element method for solving (1.1) with periodically oscillating coefficients. Then we consider the application of our method to the flow transport problem (1.1)-(1.2) (the core-plug model) with a practical random log-normal permeability. In particular, using an upscaling technique recently developed in [14, we show how the proposed mixed multiscale finite element method leads to a complete coarse grid algorithm.

Let $\Omega=(0,1) \times(0,1)$ be the unit square in $\mathbf{R}^{2}$. A uniform finite element mesh $\mathcal{M}_{h}$ is constructed by first dividing the domain $\Omega$ into $N X \times N Y$ subrectangles and then connecting the lower left and the upper right vertices of each subrectangle. In the following such a mesh is referred to the $N X \times N Y$ mesh. We divide the finite elements in the triangulation into two groups: the lower right and upper left elements. For each triangle $K$, an over-sampling element $S(K)$ is created according to whether $K$ is a lower-right element or an upper-left element as shown in Figure 1 Here we assume that the permeability field is known outside the domain $\Omega$ when the over-sampling element $S(K)$ is extended outside $\Omega$. This assumption is not a restriction for practical applications, because the permeability is generated by a geostatistical method based on the statistical characteristics, which are usually known outside the domain of interest. On the other hand, it is also possible to apply over-sampling only to the portion of the element that is inside the physical domain [18.

5.1. Accuracy of the multiscale finite element method. In this subsection, we test the over-sampling mixed multiscale finite element method by solving (2.1)(2.2) with

$$
\begin{gathered}
a_{i j}(x, x / \varepsilon)=a(x / \varepsilon) \delta_{i j}, \quad a(x / \varepsilon)=\frac{2+P \sin \left(2 \pi x_{1} / \varepsilon\right)}{2+P \sin \left(2 \pi x_{2} / \varepsilon\right)}+\frac{2+P \sin \left(2 \pi x_{2} / \varepsilon\right)}{2+P \cos \left(2 \pi x_{1} / \varepsilon\right)}, \\
f(x)=2 \pi^{2} \cos \left(\pi x_{1}\right) \cos \left(\pi x_{2}\right) \quad \text { and } \quad g(x)=0
\end{gathered}
$$


TABLE 1. Results for the over-sampling method $\varepsilon / h=0.5$ and $N=32$.

\begin{tabular}{|c|c|c|c|c|c|}
\hline $\mathrm{M}$ & $\varepsilon$ & $\mathrm{RE}_{1}$ & rate & $\mathrm{RE}_{2}$ & rate \\
\hline 8 & $1 / 16$ & $12.6155 \mathrm{E}-2$ & & $11.4035 \mathrm{E}-2$ & \\
\hline 16 & $1 / 32$ & $7.3081 \mathrm{E}-2$ & 0.7870 & $6.5842 \mathrm{E}-2$ & 0.7920 \\
\hline 32 & $1 / 64$ & $4.6645 \mathrm{E}-2$ & 0.6478 & $4.1563 \mathrm{E}-2$ & 0.6637 \\
\hline 64 & $1 / 128$ & $3.6665 \mathrm{E}-2$ & 0.3473 & $2.5297 \mathrm{E}-2$ & 0.7163 \\
\hline
\end{tabular}

TABLE 2. Results for the over-sampling method $(\varepsilon=1 / 128=0.0078125)$

\begin{tabular}{|c|c|c|c|c|c|}
\hline \multicolumn{2}{|c|}{ Mesh } & & & & \\
\hline $\mathrm{N}$ & $\mathrm{M}$ & $\mathrm{RE}_{1}$ & rate & $\mathrm{RE}_{2}$ & rate \\
\hline 128 & 16 & $6.6152 \mathrm{E}-2$ & & $6.0833 \mathrm{E}-2$ & \\
\hline 64 & 32 & $4.2149 \mathrm{E}-2$ & 0.6503 & $3.4530 \mathrm{E}-2$ & 0.8170 \\
\hline 32 & 64 & $3.6664 \mathrm{E}-2$ & 0.2011 & $2.5297 \mathrm{E}-2$ & 0.4489 \\
\hline 16 & 128 & $5.1504 \mathrm{E}-2$ & -0.4903 & $2.8311 \mathrm{E}-2$ & -0.1624 \\
\hline
\end{tabular}

where $P=1.8$. The exact solution of the test problem is unknown, and so we compare the coarse grid solutions obtained by the over-sampling multiscale method with the resolved solution $\mathbf{p}_{h}=-a(x / \varepsilon) \nabla u_{h}$, where $u_{h}$ is computed by using the standard conforming linear finite element method on the $2048 \times 2048$ mesh.

The test problem is computed on the $M \times M$ mesh by the over-sampling mixed multiscale method. For each triangle $K$, the over-sampling multiscale base functions on the over-sampling element $S(K)$ are computed on a uniform triangular mesh which divides the horizontal and vertical edges of $S(K)$ into $4 N$ subintervals, respectively. So the mesh size for solving the base functions is $1 / N M$ in each space direction.

The results of the computations are shown in Tables 1 and 2. In the tables

$$
\mathrm{RE}_{i}=\frac{\left\|\mathbf{p}_{i}^{\text {exact }}-\mathbf{p}_{i}^{\text {os }}\right\|_{0, \Omega}}{\left\|\mathbf{p}_{i}^{\text {exact }}\right\|_{0, \Omega}}, \quad i=1,2,
$$

is the relative error between the $i$-th component of the resolved flux $\mathbf{p}^{\text {exact }}$ and the corresponding component of the computed flux $\mathbf{p}^{\text {os }}$ using the over-sampling multiscale method.

Recall from Theorem 2.2 that the convergence rate is $O(\sqrt{\varepsilon}+h+\varepsilon / h)$. In Table 1 , we test the convergence of our method when $\varepsilon / h$ is fixed at 0.5 . We can see that the solution still converges as $\varepsilon$ decreases. This shows that the constant in the $\varepsilon / h$ term in the error estimate is small. We also observe that the convergence rate is better than $\sqrt{\varepsilon}$ when the resonant error term $\varepsilon / h$ is not dominant. On the other hand, the method still suffers from the secondary cell resonance as pointed out in [13]. In Table 2, we show the errors for a fixed $\varepsilon=1 / 128$. We observe that 
the errors increase as $h$ decreases, indicating the existence of the error term $\varepsilon / h$ in the error estimate. The resonant error is the strongest when $\varepsilon=h$. We remark that previous studies [18, 13] indicate that the cell resonance error is more visible for periodic coefficients, especially when $\varepsilon / h$ is a rational number. But the cell resonance error is generically small for random coefficients.

5.2. The core-plug model: local conservation property. Now we consider the application of the over-sampling mixed multiscale finite element method to the core-plug of the flow transport problem (1.1)-(1.2). Let $\Omega$ be the cross-section of the reservoir through which the flow moves. Let $\Gamma_{\text {in }}=\left\{\left(x_{1}, x_{2}\right): x_{1}=0, x_{2} \in(0,1)\right\}$, $\Gamma_{\text {out }}=\left\{\left(x_{1}, x_{2}\right): x_{1}=1, x_{2} \in(0,1)\right\}$, and $\Gamma_{N}=\left\{\left(x_{1}, x_{2}\right): x_{1} \in(0,1), x_{2}=\right.$ 0 or 1$\}$. We impose the following boundary conditions for the pressure equation (1.1):

$$
p=1 \text { on } \Gamma_{\text {in }}, \quad p=0 \text { on } \Gamma_{\text {out }}, \quad-\mathcal{K}(x) \nabla p \cdot \mathbf{n}=0 \text { on } \Gamma_{N},
$$

which represents a flow from $\Gamma_{\text {in }}$ to $\Gamma_{\text {out }}$ with no-flow boundary conditions on $\Gamma_{N}$. For the saturation equation (1.2), we impose the following initial condition and boundary condition on the inflow boundary $\Gamma_{\text {in }}$ :

$$
S(x, 0)=0 \text { in } \Omega, \quad S(x, t)=1 \text { on } \Gamma_{\text {in }} .
$$

Recall that $S$ stands for the saturation of water. Denote by $I$ the identity matrix in $\mathbf{R}^{2 \times 2}$, and assume the relative permeability tensor $\mathcal{K}(x)=k(x) I$. Geostatistical models often suggest that the logarithm of the permeability field $k(x)$ is weakly or second order stationary in space, so that the mean log permeability is constant and its covariance only depends on the relative distance of two points rather than their actual location [1], 10], [11, [21] and [31. This is to say that the permeability field is generically a log-normal random field. In this section, we will test the performance of our mixed multiscale finite element method for a random log-normal permeability field. Here we generate the random log-normal permeability field $k(x)$ by using the moving ellipse average technique [10] with the variance of the logarithm of the permeability $\sigma^{2}=1$, and the correlation lengths $l_{1}=0.3$ and $l_{2}=0.01$ in the $x_{1}$ and $x_{2}$ directions, respectively. One realization of the resulting permeability field in our numerical experiments is depicted in Figure 2.

Throughout we are interested in the fractional flow curves. A fractional flow curve $F(t)$ at time $t$ is defined as the production rate on $\Gamma_{\text {out }}$ by the following formula:

$$
F(t)=\left(\int_{0}^{1} v_{1}\left(1, x_{2}, t\right)\left(1-S\left(1, x_{2}, t\right)\right) d x_{2}\right) /\left(\int_{0}^{1} v_{1}\left(1, x_{2}, t\right) d x_{2}\right) .
$$

Here $1-S$ stands for the saturation of the produced fluid (e.g., oil), and thus $F(t)$ represents the percentage of the produced oil over water. We also use the dimensionless time PVI in our computations. One PVI is the time required to fill the whole reservoir by injecting water at $\Gamma_{\mathrm{in}}$. It is equal to [12

$$
P V I=\frac{t v_{1}^{\text {average }} L_{2}}{L_{1} L_{2}}=t v_{1}^{\text {average }},
$$

where $L_{1}$ and $L_{2}$ are the lengths of the cross-section of the reservoir in the $x_{1}$ and $x_{2}$ directions $\left(L_{1}, L_{2}\right.$ are scaled to be 1 in our simulations), $t$ is the real time and $v_{1}^{\text {average }}$ is the average velocity on $\Gamma_{\text {in }}$ in the $x_{1}$ direction. It is clear that in the unit of PVI, the time now is equal to the fraction of the water left in the whole reservoir, due to the heterogeneities of the medium. 


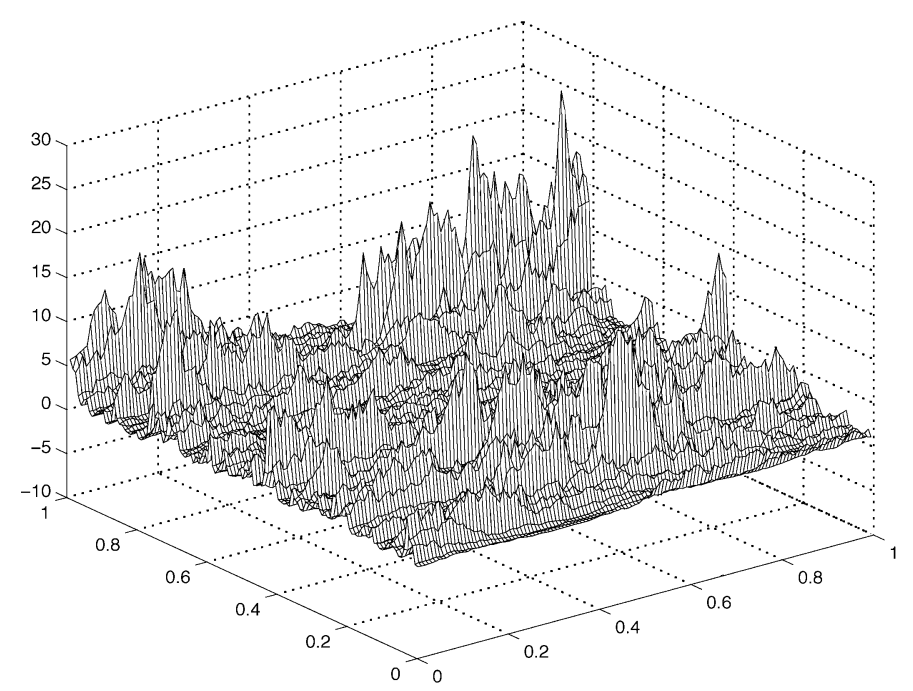

Figure 2. The random log-normal permeability field $k(x)$. The ratio of maximum to minimum is 168.65 .

The saturation equation (1.2) will be solved by an upwinding scheme in the finite element formulation; see $\left[22\right.$ and $\left[8\right.$. Given the discrete velocity field $\mathbf{v}_{h}$, for any $K \in \mathcal{M}_{h}$ with unit outer normal $\boldsymbol{\nu}$, we divide its boundary $\partial K$ into two parts:

$$
\begin{array}{ll}
\partial K_{-}=\bigcup\left\{e \subset \partial K: \mathbf{v}_{h}\left(x_{e}\right) \cdot \boldsymbol{\nu}<0\right\}, & \text { inflow, } \\
\partial K_{+}=\bigcup\left\{e \subset \partial K: \mathbf{v}_{h}\left(x_{e}\right) \cdot \boldsymbol{\nu} \geq 0\right\}, & \text { outflow. }
\end{array}
$$

Here $x_{e}$ is the midpoint of the side $e$. For any piecewise constant function $\eta_{h}$ over the mesh $\mathcal{M}_{h}$, we define its upwinding value on $\partial K$ as

$$
\tilde{\eta}_{h}= \begin{cases}\eta_{h}^{+}\left(\text {interior trace of } \eta_{h}\right) & \text { on } \partial K_{+}, \\ \eta_{h}^{-}\left(\text {exterior trace of } \eta_{h}\right) & \text { on } \partial K_{-},\end{cases}
$$

and assume $\eta_{h}^{-}=1$ on $\partial K_{-} \cap \Gamma_{\text {in }}$. Let $\tau$ be the time step and $t^{n}=n \tau, n \geq 1$. Then, denoting by $S_{K}^{n}$ the approximation of the water saturation in $K$ at time $t^{n}$, we use the following upwinding scheme to solve (1.2):

$$
\frac{S_{K}^{n+1}-S_{K}^{n}}{\tau}+\int_{\partial K}\left(\mathbf{v}_{h} \cdot \boldsymbol{\nu}\right) \tilde{S}_{K}^{n} d s=0 \quad \forall K \in \mathcal{M}_{h} .
$$

We note that in the scheme (5.1) only the normal components of the discrete velocity on the sides of each element $K$ are required. For the mixed finite element methods solving (1.1), such normal components are directly computed as part of the method. In the following simulations, the "exact" fractional flow curve is referred to the one computed by solving (1.1) by the lowest order Raviart-Thomas finite element method and (1.2) by the scheme (5.1) on the fine $1024 \times 1024$ mesh.

Our first experiment in this subsection is designed to show the importance of the local conservation property in the flow transport simulations. Let $\mathbf{v}_{h}$ be the approximation of the Darcy velocity $\mathbf{v}=-k(x) \nabla p$; then the local conservation 
property means

$$
\int_{\partial K}\left(\mathbf{v}_{h} \cdot \boldsymbol{\nu}\right) d s=0 \quad \forall K \in \mathcal{M}_{h}
$$

This is an important property, because if it is violated it would introduce unphysical numerical sources or sinks. It is clear that (5.2) is always satisfied when the mixed finite element method is used to solve (1.1). In this experiment we first solve the pressure equation (1.1) by using a standard conforming linear finite element method on the $1024 \times 1024$ mesh to get $p_{h}$, and then we recover the normal components of the velocity by two different methods. For any $K \in \mathcal{M}_{h}$, in the first method we take $\left.\mathbf{v}_{h} \cdot \boldsymbol{\nu}\right|_{\partial K}$ as the interior trace of $\mathbf{v}_{h}=-k(x) \nabla p_{h}$. The resulting fractional flow curve is depicted in Figure 3(a). We note that in this method the normal components of the velocity are in general discontinuous across the interelement boundaries, but the local conservation property (5.2) is satisfied since $k(x)$ is constant on each element $K$ and so is $\nabla p_{h}$. In the second method, we take $\mathbf{v}_{h} \cdot \boldsymbol{\nu}$ on any side $e \subset \partial K$ as the average of its values in two adjacent elements. The resulting fractional flow curve is shown in Figure 3(b). We can see clearly that the fractional flow curve obtained from the second method deviates significantly from the solid line obtained from the "exact" solution. Note that in this method the normal components are continuous across the interelement boundaries, but the local conservation property is violated. This shows that the local conservation property is more important than the continuity of the normal components. Moreover, we observe that the fractional flow curve computed by the first method is in good agreement with the "exact" one obtained by using the mixed method on the fine $1024 \times 1024$ mesh. We remark that the mixed finite element method involves the computation of the solution of a discrete problem which has many more degrees of freedom than solving the pressure equation (1.1) using a linear finite element method. Thus, when one can afford to solve the whole system (1.1)-(1.2) on a fine mesh, instead of using the mixed finite element method, one should use the linear finite element method to solve the pressure equation (1.1) and then generate the velocity field by the first method mentioned above. However, we remark that the linear finite element method is a special case. If one uses higher order displacement finite element methods to solve (1.1), there are no obvious ways to recover the velocity field such that the local conservation property (5.2) is satisfied.

Next we compare the performance of the mixed multiscale finite element method and the displacement multiscale finite element method 18. One important feature of the multiscale finite element method is its ability to reconstruct locally the finescale velocity field from the coarse grid pressure solver. The key idea is to use the multiscale base functions to interpolate the fine grid solution. To demonstrate that we can accurately capture the correct small-scale velocity field, we first solve the pressure equation (1.1) on a coarse $64 \times 64$ mesh and use the multiscale finite element basis functions to reconstruct the velocity field on the fine $1024 \times 1024$ mesh. We then use this locally reconstructed fine grid velocity field to solve the saturation equation (1.2) on the fine $1024 \times 1024$ mesh. In Figure 4(a), the resulting fractional flow curve is compared with the "exact" fractional flow curve which is obtained by solving the pressure and the saturation equations on the fine $1024 \times 1024$ mesh using the mixed finite element method. As we can see from Figure 4(a), the two fractional flow curves agree very well. 

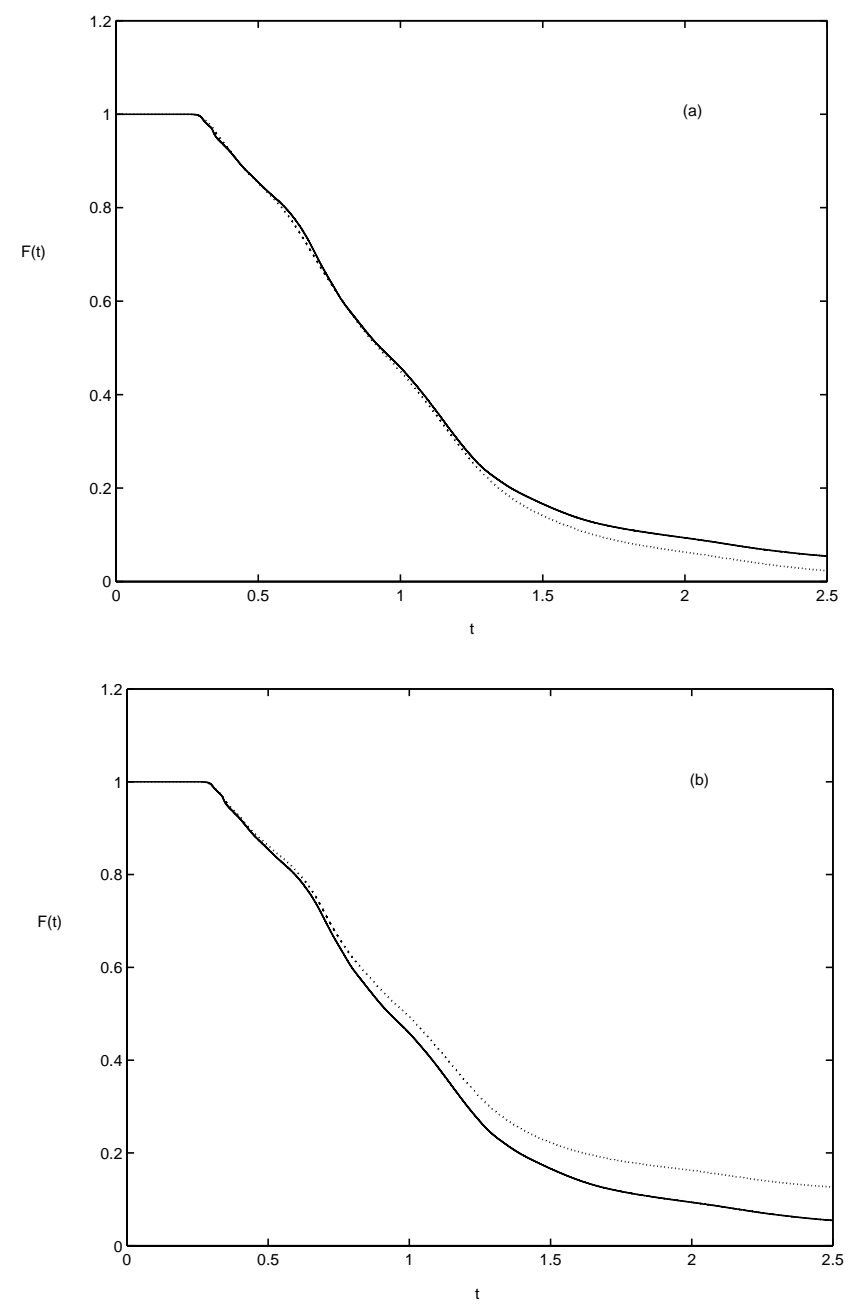

Figure 3. Comparison of two different methods recovering the normal components of the velocity from piecewise linear pressure $p_{h}$. Each solid line is the "exact" fractional flow curve using the mixed finite element method solving the pressure equation. (a) The dotted line is the fractional flow curve obtained from the first method of local velocity recovery, which satisfies the local conservation property. (b) The dotted line is the fractional flow curve obtained from the second method of local velocity recovery, which violates the local conservation property.

We then solve the pressure equation (1.1) by the displacement over-sampling multiscale finite element method on a coarse $64 \times 64$ mesh and use the displacement multiscale finite element bases to reconstruct the velocity field on the fine mesh. Again the saturation equation (1.2) is solved on the fine $1024 \times 1024$ mesh. The resulting fractional flow curve and the "exact" fraction curve are shown in Figure 4 (b). Due to the violation of the local conservation property, the displacement 

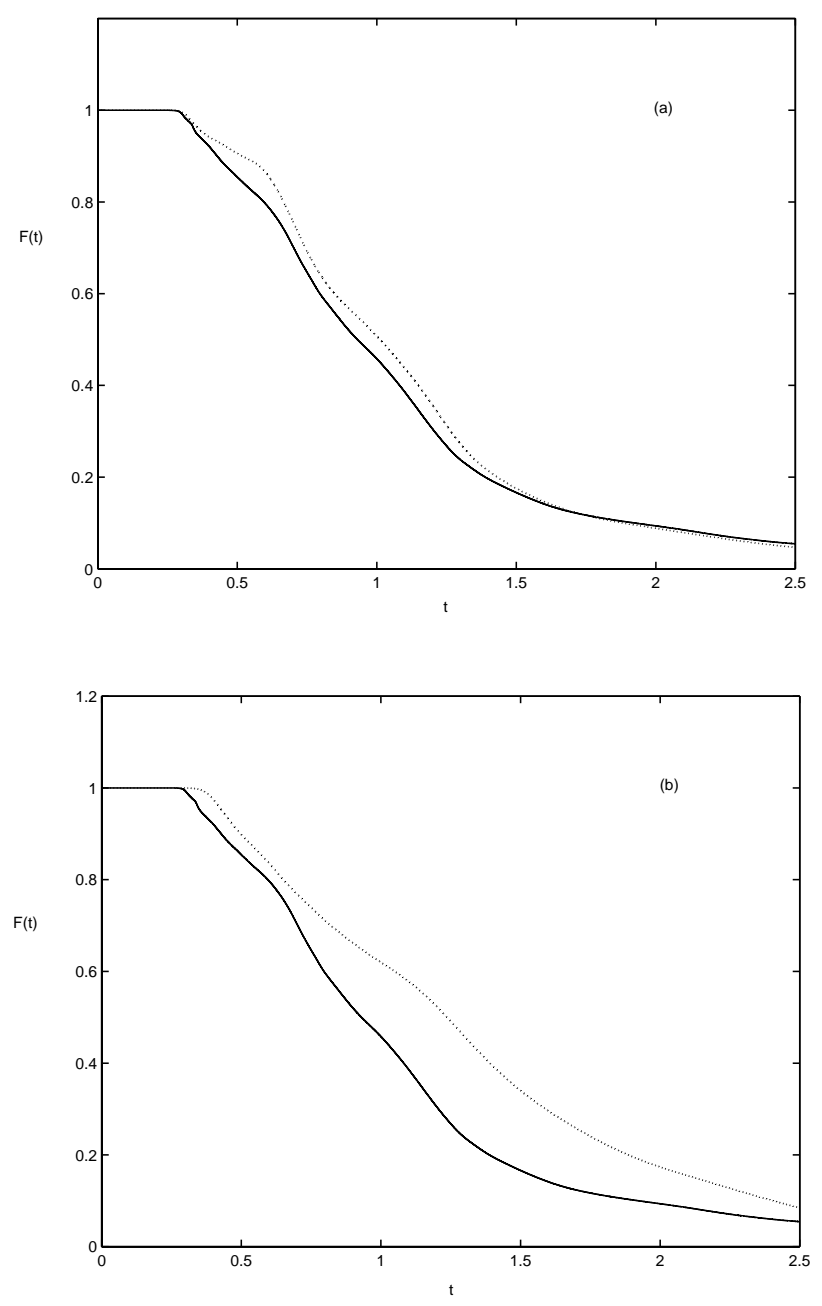

Figure 4. Comparison of the mixed multiscale finite element method and the displacement multiscale finite element method. Each solid line is the "exact" fractional flow curve using the mixed finite element method to solve the pressure equation. (a) The dotted line is the fractional flow curve using the velocity by the mixed multiscale finite element method. (b) The dotted line is the fractional flow curve using the velocity recovered from the displacement multiscale finite element method.

multiscale finite element method begins to deviate significantly from the "exact" fractional flow curve after some time (beyond $t=0.6$ ). This numerical example demonstrates that the over-sampling mixed multiscale finite element method provides a better way to locally reconstruct the fine grid velocity field which is suitable for long-time computations of the saturation field. This is an important consideration from a practical viewpoint. 
5.3. The core-plug model: a coarse mesh algorithm. Now we describe how the proposed mixed multiscale finite element can be combined with the existing upscaling technique for the saturation equation (1.2) to get a complete coarse grid algorithm for the problem (1.1)-(1.2). The numerical upscaling of the saturation equation has been under intensive study in the literature, see [11, [14, 12, and 21]. Here, we use the upscaling method proposed in [14] and [12] to design an overall coarse grid model for the problem (1.1)-(1.2). The work of [14] for upscaling the saturation equation involves a moment closure argument. The velocity and the saturation are separated into a local mean quantity and a small-scale perturbation with zero mean. For example, the Darcy velocity is expressed as $\mathbf{v}=\mathbf{v}_{0}+\mathbf{v}^{\prime}$ in (1.2), where $\mathbf{v}_{0}$ is the average of the velocity $\mathbf{v}$ over each coarse element and $\mathbf{v}^{\prime}=\left(\mathbf{v}_{1}^{\prime}, \mathbf{v}_{2}^{\prime}\right)$ is the deviation of the fine-scale velocity from its coarse-scale average. After some manipulations, an average equation for the saturation $S$ can be derived as follows [14]:

$$
\frac{\partial S}{\partial t}+\mathbf{v}_{0} \cdot \nabla S=\frac{\partial}{\partial x_{i}}\left(D_{i j}(x, t) \frac{\partial S}{\partial x_{j}}\right),
$$

where the diffusion coefficients $D_{i j}(x, t)$ are defined by

$$
D_{i i}(x, t)=\left\langle\left|\mathbf{v}_{i}^{\prime}(x)\right|\right\rangle L_{i}^{0}(x, t), \quad D_{i j}(x, t)=0, \quad \text { for } i \neq j,
$$

$\left\langle\left|\mathbf{v}_{i}^{\prime}(x)\right|\right\rangle$ stands for the average of $\left|\mathbf{v}_{i}^{\prime}(x)\right|$ over each coarse element and $L_{i}^{0}(x, t)$ is the length of the coarse grid streamline in the $x_{i}$ direction which starts at time $t$ at point $x$, i.e.,

$$
L_{i}^{0}(x, t)=\int_{0}^{t} y_{i}(s) d s,
$$

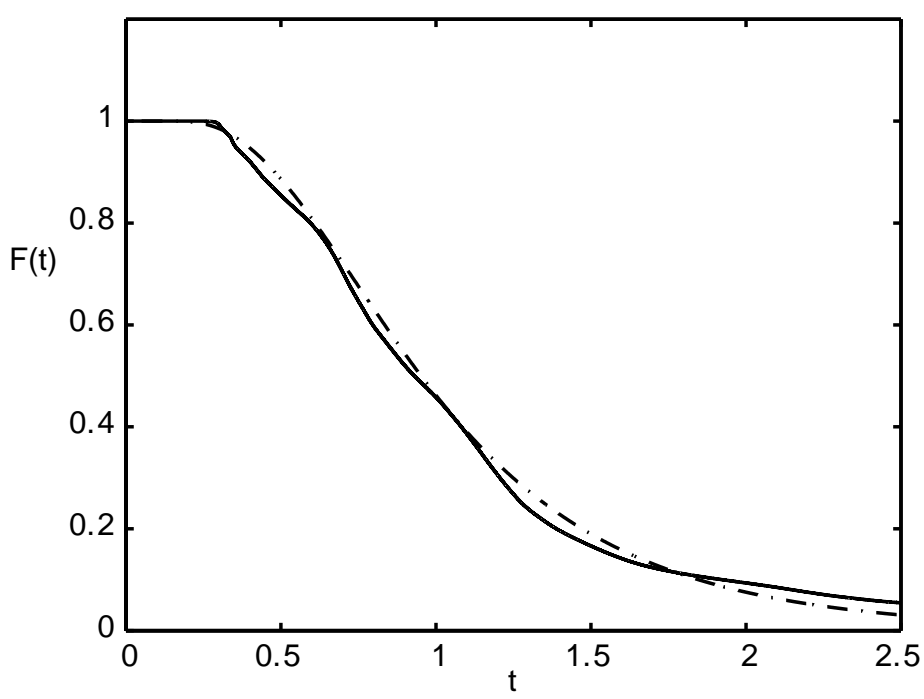

Figure 5. The accuracy of the coarse grid algorithm. The solid line is the "exact" fractional flow curve using the mixed finite element method for solving the pressure equation. The slash-dotted line is the fractional flow curve using the above coarse grid algorithm. 
where $y(s)$ is the solution of the following system of ODEs:

$$
\frac{d y(s)}{d s}=\mathbf{v}_{0}(y(s)), \quad y(t)=x .
$$

Note that the hyperbolic equation (1.2) is now replaced by a convection-diffusion equation. The convection-dominant parabolic equation (5.3) is solved by the characteristic linear finite element method [9], 26] in our simulation. The flow transport model (1.1)-(1.2) is solved in the coarse grid as follows:

1. Solve the pressure equation (1.1) by the over-sampling mixed multiscale finite element method and obtain the fine-scale velocity field using the multiscale basis functions.

2. Compute the coarse grid average $\mathbf{v}_{0}$ and the fine-scale deviation $\left\langle\left|\mathbf{v}_{i}^{\prime}(x)\right|\right\rangle$ on the coarse grid.

3. At each time step, solve the convection-diffusion equation (5.3) by the characteristic linear finite element method on the coarse grid in which the lengths $L_{i}^{0}(x, t)$ of the streamline are computed for the center of each coarse grid element.

The mixed multiscale finite element method can be readily combined with the above upscaling model for the saturation equation. The local fine grid velocity $\mathbf{v}^{\prime}$ will be constructed from the multiscale finite element base functions. The main cost in the above algorithm lies in the computation of multiscale bases, which can be done a priori and completely in parallel. This algorithm is particularly attractive when multiple simulations must be carried out due to the change of boundary and source distribution, as is often the case in engineering applications. In such situation, the cost of computing the multiscale base functions is just overhead. Moreover, once these base functions are computed, they can be used for subsequent time integration of the saturation. Because the evolution equation is now solved on a coarse grid, a larger time step can be used. This also offers additional computational saving. For many oil recovery problems, due to the excessively large fine grid data, upscaling is a necessary step before performing many simulations and realizations on the upscaled coarse grid model. If one can coarsen the fine grid by a factor of 10 in each dimension, the computational saving of the coarse grid model over the original fine model could be as large as a factor 10,000 (three space dimensions plus time).

We perform a coarse grid computation of the above algorithm on the coarse $64 \times 64$ mesh. The fractional flow curve using the above algorithm is depicted in Figure 5. It gives excellent agreement with the "exact" fractional flow curve. The contour plots of the saturation $S$ at times $t=0.25$ and $t=0.5$ are depicted in Figure 6. The contour plots of the saturation $S$ on the fine $1024 \times 1024$ mesh at times $t=0.25$ and $t=0.5$ computed by using the "exact" velocity field are displayed in Figure 7 We observe that the contour plots in Figure 6 approximate the "exact" ones in Figure 7 in accuracy, but the sharp oil/water interfaces in Figure 7 are smeared out. This is due to the parabolic nature of the upscaled equation (5.3). We have also performed many other numerical experiments to test the robustness of this combined coarse grid model. We found that for permeability fields with strong layered structure, the above coarse grid model is very robust. The agreement with the fine grid calculations is very good. We are currently working towards a qualitative and quantitative understanding of this upscaling model. It is 

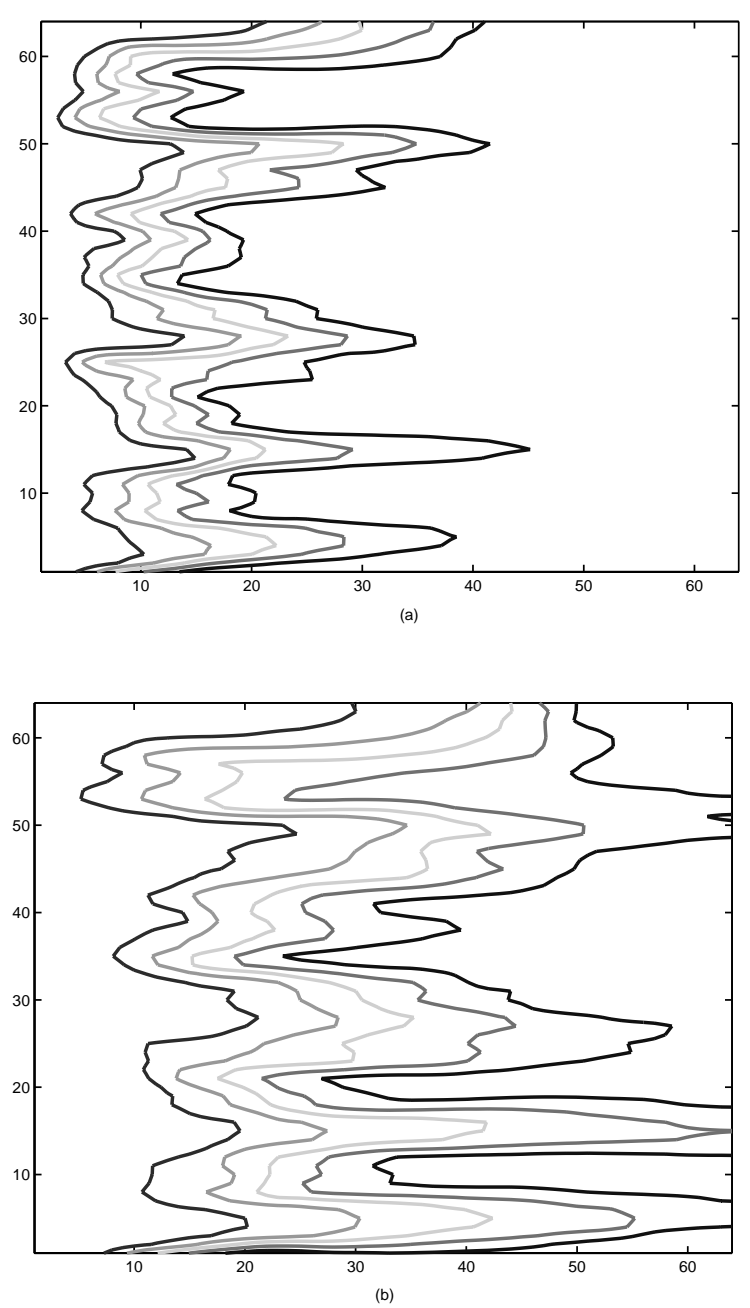

Figure 6 . The contour plots of the saturation $S$ computed using the upscaled model on a $64 \times 64$ mesh at time $t=0.25$ (left) and $t=0.5$ (right)

likely that one can justify this upscaling model for a periodic layered permeability field.

Finally, we remark that the upscaling equation (5.3) uses small-scale information $\mathbf{v}^{\prime}$ of the velocity field to define the diffusion coefficients. This information can be constructed locally through the mixed multiscale basis functions. This is an important property of our multiscale finite element method. It is clear that solving directly the homogenized pressure equation

$$
\operatorname{div}\left(\mathcal{K}^{*}(x) \nabla p^{*}\right)=0
$$

will not provide such small-scale information. On the other hand, whenever one can afford to resolve all the small-scale features by a fine grid, one can use fast linear solvers, such as multigrid methods, to solve the pressure equation (1.1) on the fine mesh. From the fine grid computation, one can easily construct the average velocity 

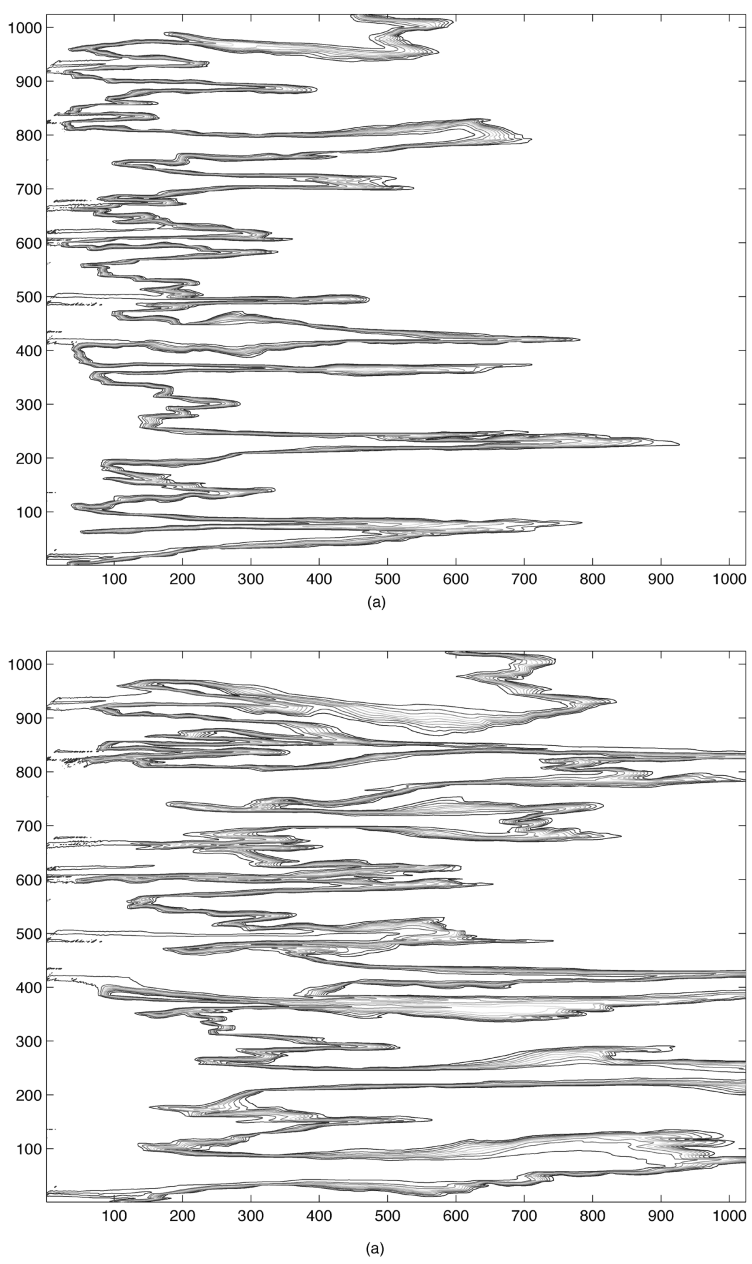

Figure 7 . The contour plots of the saturation $S$ computed on the fine $1024 \times 1024$ mesh using the "exact" velocity field at time $t=0.25$ (left) and $t=0.5$ (right)

$\mathbf{v}_{0}$ and its deviation $\mathbf{v}^{\prime}$. However, when multiple simulations must be carried out due to the change of boundary conditions, the pressure equation (1.1) will then have to be solved again on the fine mesh. The multiscale finite element method only solves the pressure equation once on a coarse mesh, and the fine grid velocity can be constructed locally through the finite element bases. This is the main advantage of our mixed multiscale finite element method. This process becomes more difficult for a nonlinear two-phase flow, due to the dynamic coupling between the pressure and the saturation. We are now investigating the possibility of upscaling the two-phase flow by using multiscale finite element base functions that are constructed from the one-phase flow (time-independent). In this case, we need to provide corrections to the pressure equation to account for the scale interaction near the oil/water interface. 
Acknowledgments. We wish to thank Dr. Yalchin R. Efendiev for many inspiring discussions and for providing us the code generating the log-normal permeability field. We would also like to thank Dr. Hector Ceniceros for his valuable comments on our original manuscript, and the referee for his careful reading and constructive comments.

\section{REFERENCES}

1. T. Arbogast, Numerical subgrid upscaling of two-phase flow in porous media, TICAM Report 99-30, University of Texas at Austin, 1999.

2. M. Avellaneda and F.-H. Lin, Compactness methods in the theory of homogenization, Comm. Pure Appl. Math. 40 (1987), 803-847. MR 88i:35019

3. I. Babuska, G. Caloz, and E. Osborn, Special finite element methods for a class of second order elliptic problems with rough coefficients, SIAM J. Numer. Anal. 31 (1994), 945-981. MR 95g:65146

4. A. Bensoussan, J.L. Lions, and G. Papanicolaou, Asymptotic Analysis for Periodic Structures, North-Holland, Amsterdam, 1978. MR 82h:35001

5. F. Brezzi and M. Fortin, Mixed and Hybrid Finite Element Methods, Springer, New York, 1991. MR 92d:65187

6. F. Brezzi, L.P. Franca, T.J.R. Hughes, and A. Russo, $b=\int g$, Comput. Methods Appl. Mech. Engrg. 145 (1997), 329-339. MR 98g:65086

7. Z. Chen, Mixed finite element methods for a dynamical Ginzburg-Landau model in superconductivity, Numer. Math. 76 (1997), 323-353. MR 98d:65139

8. Z. Chen and Q. Du, An upwinding mixed finite element method for a mean field model of superconducting vortices, RARIO Math. Model. Numer. Anal. 34 (2000), 687-706. MR 2001f:65133

9. J. Douglas, Jr. and T.F. Russell, Numerical methods for convection-dominated diffusion problem based on combining the method of characteristics with finite element or finite difference procedures, SIAM J. Numer. Anal. 19 (1982), 871-885. MR 84b:65093

10. L.J. Durlofsky, Numerical calculation of equivalent grid block permeability tensors for heterogeneous porous media, Water Resources Research 27 (1991), 699-708.

11. L.J. Durlofsky, R.C. Jones, and W.J. Milliken, A nonuniform coarsening approach for the scale-up of displacement processes in heterogeneous porous media, Adv. Water Resources, 20 (1997), 335-347.

12. Y.R. Efendiev, The Multiscale Finite Element Method and its Applications, Ph.D. thesis, California Institute of Technology, 1999.

13. Y.R. Efendiev, T.Y. Hou, and X.H. Wu, The convergence of nonconforming multiscale finite element methods, SIAM J. Numer. Anal. 37 (2000), 888-910. MR 2002a:65176

14. Y.R. Efendiev, L.J. Durlofsky, and S.H. Lee, Modeling of subgrid effects in coarse scale simulations of transport in heterogeneous porous media, Water Resources Research 36 (2000), 2031-2041.

15. D. Gilbarg and N.S. Trudinger, Elliptic Partial Differential Equations of Second Order, Springer-Verlag, Berlin, 1983. MR 86c:35035

16. V. Girault and P.-A. Raviart, Finite Element Methods for Navier-Stokes Equations, Springer, Berlin, 1986. MR 88b:65129

17. P. Grisvard, Elliptic Problems on Nonsmooth Domains, Pitman, Boston, 1985. MR 86m:35044

18. T.Y. Hou and X.H. Wu, A multiscale finite element method for elliptic problems in composite materials and porous media, J. Comput. Phys. 134 (1997), 169-189. MR 98e:73132

19. T.Y. Hou, X.H. Wu, and Z. Cai, Convergence of a multiscale finite element method for elliptic problems with rapidly oscillating coefficients, Math. Comp. 68 (1999), 913-943. MR 99i:65126

20. V.V. Jikov, S.M. Kozlov, and O.A. Oleinik, Homogenization of Differential Operators and Integral Functionals, Springer, Berlin, 1994. MR 96h:35003b

21. P. Langlo and M.S. Espedal, Macrodispersion for two-phase, immiscible flow in porous media, Adv. Water Resources 17 (1994), 297-316. 
22. P. Lesaint and P.-A. Raviart, "On a Finite Element Method for Solving the Neutron Transport equation", in Mathematical Aspects of the Finite Element Method in Partial Differential Equations, ed. by C. de Boor, Academic Press, New York, 1974. MR 58:31918

23. G.M. Lieberman, Oblique derivative problems in Lipschitz domains II. Discontinuous boundary data, J. Reine Angew. Math. 389 (1988), 1-21. MR 89h:35094

24. J.F. McCarthy, Comparison of fast algorithms for estimating large-scale permeabilities of heterogeneous media, Transport in Porous Media, 19 (1995), 123-137.

25. S. Moskow and M. Vogelius, First order corrections to the homogenized eigenvalues of a periodic composite medium. A convergence proof, Proc. Royal Soc. Edinburgh A 127 (1997), 1263-1299. MR 99g:35018

26. O. Pironneau, On the transport-diffusion algorithm and its application to the Navier-Stokes equations, Numer. Math. 38 (1982), 309-332. MR 83d:65258

27. P. Raviart and J. Thomas, "A mixed element method for 2nd order elliptic problems", in Mathematical Aspects of the Finite Element Method, Lecture Notes on Mathematics 606, Springer, Berlin, 1977. MR 58:3547

28. T.F. Russell and M.F. Wheeler, "Finite element and finite difference methods for continuous flows in porous media", in The Mathematics of Reservoir Simulation, R.E. Ewing, ed., SIAM, Philadelphia, 1983.

29. L. Tartar, "Nonlocal Effect Induced by Homogenization", in PDEs and Calculus of Variations, F. Columbini, ed., Birkhäuser Publ., Boston, 1989.

30. T.C. Wallstrom, S.L. Hou, M.A. Christie, L.J. Durlofsky and D.H. Sharp, Accurate scale up of two phase flow using renormalization and nonuniform coarsening, Computational Geosciences 3 (1999), 69-87.

31. S. Verdiere and M.H. Vignal, Numerical and theoretical study of a dual mesh method using finite volume schemes for two-phase flow problems in porous media, Numer. Math. 80 (1998), 601-639. MR 99g:76117

32. P.M. De Zeeuw, Matrix-dependent prolongation and restrictions in a blackbox multigrid solver, J. Comput. Appl. Math. 33 (1990), 1-27. MR 92e:65152

LSEC, Institute of Computational Mathematics, Chinese Academy of Sciences, BeiJing 100080, Peoples Republic of China

E-mail address: zmchen@lsec.cc.ac.cn

Applied Mathematics, California Institute of Technology, Pasadena, California 91125 .

E-mail address: hou@acm.caltech.edu 